\title{
Improved XFEM: accurate and robust dynamic crack growth simulation
}

\author{
Longfei Wen ${ }^{\mathrm{a}} \quad$ Rong Tian ${ }^{\mathrm{b}^{*}}$ \\ a Institute of Computing Technology, Chinese Academy of Sciences, Beijing 100190, China \\ ${ }^{\mathrm{b}}$ Software center for high performance numerical simulation, Institute of Applied Physics and \\ Computational Mathematics, Beijing 100088, China
}

\begin{abstract}
The extended finite element method (XFEM) is widely accepted in academy as the major technique for crack analysis. Starting from 2009, commercial codes started to use this technique for crack analysis, singling the mature and acceptance of the technique in industries. However, the direct extension of the singular tip enrichment of XFEM, the core of the method, to dynamic crack growth simulation has long been a difficulty due to: (a) elevated bad conditioning as crack propagating, (b) extra-dof dynamics and energy inconsistency, and (c) "null" critical time step size and optimal mass lumping at crack tip. Based on an extra-dof-free partition of unity enrichment technique (R. Tian. Comput. Methods Appl. Mech. Engrg. 266 (2013) 1-22), we have improved XFEM through a crack tip enrichment without extra dof $(R$. Tian, L. Wen. Comput. Methods Appl. Mech. Engrg. 285 (2015) 639-658.). This paper is to answer the question whether the improved XFEM can also be easily extended to dynamic problems. Numerical tests show that the new XFEM is not only straightforward in implementation in dynamic problems, also provides the most accurate dynamic SIF in the benchmark problems and is orders of magnitude faster with an iterative solver.
\end{abstract}

Keywords: XFEM, extra-dof free enrichment, dynamic crack growth, partition of unity

\section{Introduction}

The modelling of discontinuities such as macro- and micro-cracks is a problem of great importance in the simulation of failure. The classical way to model discontinuities is re-meshing which is to align the mesh so that the discontinuities are coincident with element edges. However, this way of simulation is quite difficult to apply in crack growth, because the mesh must be changed to conform to the crack geometry at every time step, and significant errors in the projection of the time dependent data around the crack can be introduced by re-meshing.

The extended finite element method (XFEM) [1]-[3] has been developed to overcome this long-standing difficulty. Tremendous successes have been made. Nowadays, XFEM becomes a dominant numerical technique for problems concerning discontinuities, in particular crack propagation. In 2001, XFEM was firstly applied to arbitrary discontinuities problems [3], then was used for arbitrary three-dimensional

\footnotetext{
* Corresponding author. Tel.: +86 1061935646.

E-mail Address: tian_rong@iapcm.ac.cn (R. Tian).
} 
cracks by Gravouil et al.[4]. It was further extended to many applications like composites [5], incompressible solids [6], magnetoelectroelastic solids [7], ductile cracks [8], and fatigue cracks [9]. Two recent excellent review papers by Belytschko and his collaborators [10][11] and the XFEM monograph [49][50] can be referred for further details.

Since 2010, several commercial software packages such as ABAQUS [12], ANSYS, ASTER and ALTAIR have officially supported XFEM as a new tool for crack analysis, signaling XFEM is accepted also in industries.

Although it achieved unprecedented success in steady/time-independent problems, the method's extension to dynamic crack growth simulation has long been a challenge, due to three aspects of issues:

(1) Elevated bad conditioning;

(2) Extra-dof dynamics and energy inconsistency;

(3) "Null" critical time step size and optimal mass lumping at crack tip.

Although XFEM is enriched only locally, it suffers from the common stability issue called linear dependence due to the nature of partition of unity approximation (PU) [13]-[15], which exhibits ill-conditioned global (stiffness) matrices [16]-[21]. Numerical tests [39][40] showed that the conditioning number of the global matrix of XFEM for the geometrical refinement, i.e., the constant size of enriched tip domain, increases as $h^{-6}$, where $h$ is the uniform mesh size, in contrast to $h^{-2}$ in the standard FEM. Due to the severely bad conditioning, convergence with an iterative solver is slow or fails in an implicit time integration. The poor convergence is magnified further by an increasing number of extra nodal dofs in dynamic crack growth analysis.

As a remedy for the bad conditioning in a broad context of the partition of unity approximation, a stable GFEM has been proposed in 2012 in 1D, followed by an extension to high dimensions in 2013 [20][21]. The key idea of the stable GFEM is to subtract the standard FE approximation from the local approximation. As a result, the conditioning of global matrices recovers the property of $h^{-2}$ [20]. The stabilization technique is also applied to XFEM [41]. But the stable GFEM still contains extra dofs and may encounter the similar problems to XFEM in dynamics.

The second issue, the conservative energy transfer, has been and still is the most daunting issue in XFEM in time dependent problems. The issue arises in the situation when a crack tip leaves the element and its partial nodes turn from a tip-enriched node to a Heaviside enriched one. Due to the change in the enrichment functions, the nodal translational dofs, velocities, accelerations have to be changed. However, if they are simply discarded and switched to Heaviside enriched extra dofs, the energy would not be properly transferred to the next time step, leading to unaccepted accuracy loss. This means the extension to dynamic analysis is not as straightforward as that in static problems. This has been a very frustrating issue in the history of XFEM.

In order to circumvent the issue, the early suggested remedy is to discard the tip enrichment and to keep only the Heaviside enrichment in dynamic crack growth simulation [22]. However, the core merit of the singular enrichment of XFEM, which is remarkably successful in static analysis, has to be compromised [25]. Although the XFEM with only Heaviside enrichment shows a certain success in dynamic crack growth simulation, the attempt at maintaining singular enrichment for dynamic crack growth analysis never stops. The first major success may be traced back to Réthore et al. [28]-[30]. The basic idea of the suggested remedy is that: only to append and never to delete any extra dof, including generalized nodal terms like velocity and acceleration. For example, for a given tip element, a new set of extra dofs for singular enrichment are added for each new tip position if the crack is propagating within element and the 
new extra dofs for Heaviside enrichment are added finally when the crack tip leaves the element. The shape function associated with each set of extra dofs records the history of crack propagation. It can be verified that this approach guarantees the energy represented or "transferred" exactly at the beginning of the next step. The payoff is this approach introduces a considerable number of extra dofs to a node.

The third issue is mainly relevant to explicit dynamic analysis. The optimal mass lumping of extra dofs in XFEM has been an open issue. For this reason, the consistent mass matrix has been employed in the early literature for dynamic crack problems [22][31]. Later on, an "optimal" mass lumping technique was found by Menouillard et al. [23][24] for a Heaviside enriched element and later on the technique was extended in a more general sense to any enrichment functions such as the crack tip enrichment by Elguedj et al. [32] in 2009.

Despite the above successes, XFEM still cannot achieve the same level of accuracy in a dynamic crack growth simulation as that in a stationary crack one. For crack propagating under dynamic loading, the dynamic stress intensity factor obtained by XFEM shows "unacceptable" oscillation [23]-[26][30][32]-[34]. Continuous attempts have been made at understanding and eliminating the oscillation: Menouillard and Belytschko [27] coupled XFEM with meshfree approximation and observed that the meshfree approximation helps but not too much with reducing the oscillation. Later, Menouillard et al. (2010a) [25] studied several different enrichment strategies for dynamic crack propagation and observed that the oscillation can be weakened somewhat by enlarging the tip-enrichment domain; Liu et al. [34] combined a spectral element method with the singular enrichment of XFEM and observed that the high order spectral approximation reduces to some extents the oscillation; Menouillard and Belytschko [26] discarded the singular enrichment for crack tip, used instead the cohesive element technique, and introduced a force correction scheme to smooth out the sudden release of a tip element to a cut element. The numerical tests reported showed that the cohesive element approach together with the force correction reduced the oscillation and achieved good accuracy.

Nevertheless, to the best knowledge of the authors', how the XFEM with the crack tip singular enrichment delivers good accuracy in dynamic crack growth simulation remains unsolved. We reasoned that the extra dof may be the very root of all issues in the current GFEM/XFEM and hence have developed an extra-dof free PU approximation [38] and then an improved XFEM [39][40]. Keeping in mind that the direct extension of XFEM to dynamic crack growth simulation has long been a difficulty, this paper tends to answer the question whether the improved XFEM is straightforward in dynamic crack growth simulation in implementation.

\section{Improved XFEM (iXFEM)}

\subsection{Extra-doffree PU approximation}

Let $\Omega^{\text {tip }}$ be a sub-domain containing a crack tip. All nodes in $\Omega^{\text {tip }}$ are enriched. Define $\mathrm{P}_{i}^{r}$ be a nodal patch of node $i$. The nodal patch is composed of nodes surrounding node $i$ within an influence radius $r$. The patch size $r$ is either the size of nodal support or simply the radius of an influence circle at node $i$. Node $i$ is referred as "patch star". Let $I_{i}=\left\{k \in I: x_{k} \in \mathrm{P}_{i}^{r}\right\}$ be the node set on $\mathrm{P}_{i}^{r}, \quad J_{i}=\left\{k \in I: x_{i} \in \mathrm{P}_{k}^{r}\right\}$ be the nodal patch set containing node $i$. The two sets are identical in terms of nodal indexes, but they are different in logic. 
On the patch $\mathrm{P}_{i}^{r}$, a local least-squares approximation of field function $u_{i}(\mathbf{x})$ is constructed as

$$
\begin{gathered}
u_{i}^{\mathrm{loc}}(\mathbf{x})=\mathbf{p}^{\mathrm{T}}(\mathbf{x}) \mathbf{a}, \mathbf{x} \in \mathrm{P}_{i}^{r} \\
u_{i}^{\mathrm{loc}}\left(\mathbf{x}_{i}\right)=u_{i}
\end{gathered}
$$

where $\mathbf{p}(\mathbf{x})$ is the basis vector and $\mathbf{a}$ is the vector of unknown coefficients, the local approximation is required to interpolate at the "patch star" node, and at only this one point. For the patch containing the crack tip, the basis $\mathbf{p}(\mathbf{x})$ is chosen as

$$
\begin{aligned}
& \mathbf{p}(\mathbf{x})=\left[p_{1}(\mathbf{x}), p_{2}(\mathbf{x}), \ldots, p_{m}(\mathbf{x})\right]^{\mathrm{T}} \\
&=\left[1, \frac{x-x_{i}}{c h}, \frac{y-y_{i}}{c h}, f_{\alpha}(r, \theta)-f_{\alpha}\left(r_{i}, \theta_{i}\right)\right]^{\mathrm{T}} \\
& f_{\alpha}(r, \theta)(x) \in\left\{\sqrt{r} \cos \left(\frac{\theta}{2}\right), \sqrt{r} \sin \left(\frac{\theta}{2}\right), \sqrt{r} \sin \left(\frac{\theta}{2}\right) \sin \theta, \sqrt{r} \cos \left(\frac{\theta}{2}\right) \sin \theta\right\}
\end{aligned}
$$

where $c h$ denotes the patch size, $h$ is mesh size and $c$ is a constant, $c=2$ or 3 .

In order to construct a least-squares approximation passing the patch star, the following constrained form of discrete $\mathrm{L}_{2}$ error norm is used,

$$
J=\frac{1}{2} \sum_{k \in I_{i}}\left(\mathbf{p}^{\mathrm{T}}\left(\mathbf{x}_{k}\right) \mathbf{a}-u_{k}\right)^{2}+\lambda\left(\mathbf{p}^{\mathrm{T}}\left(\mathbf{x}_{i}\right) \mathbf{a}-u_{i}\right)
$$

where the Lagrange multiplier $\lambda$ is used to enforce the condition (2), i.e.,

$$
u_{i}^{\text {loc }}(\mathbf{x})=\mathbf{p}^{\mathrm{T}}\left(\mathbf{x}_{i}\right) \mathbf{a} \equiv u_{i}
$$

Minimizing the $\mathrm{L}_{2}$ norm $(\partial J / \partial \mathbf{a}=0, \partial J / \partial \lambda=0)$ and cancelling $\lambda$ lead to

$$
\begin{gathered}
u_{i}^{\mathrm{loc}}(\mathbf{x})=\sum_{k \in I_{i}} \phi_{k}^{i}(\mathbf{x}) u_{k} \\
\phi_{k}^{i}(\mathbf{x})=\mathbf{p}^{\mathrm{T}}(\mathbf{x})\left(\mathbf{A}^{-1} \mathbf{p}_{k}-\frac{1}{\mathbf{A}_{11}^{-1}} \mathbf{A}_{(1)}^{-1} \mathbf{A}_{(1)}^{-\mathrm{T}} \mathbf{p}_{k}+\frac{1}{\mathbf{A}_{11}^{-1}} \mathbf{A}_{(1)}^{-1} \delta_{i^{*} k}\right) \\
\mathbf{A}=\sum_{k \in I_{i}} \mathbf{p}_{k} \mathbf{p}_{k}^{\mathrm{T}}
\end{gathered}
$$

where the moment matrix $\mathbf{A}$ is a constant matrix on patch, $\mathbf{A}_{(1)}^{-1}$ is the first column of $\mathbf{A}^{-1}, \mathbf{A}_{11}^{-1}$ is the first element of $\mathbf{A}_{(1)}^{-1}$, and $\delta$ is the Kronecker delta. It can be verified that $\phi_{i}^{i}\left(\mathbf{x}_{i}\right) \equiv 1$.

Substitute (6) for the nodal unknown $u_{i}$ in the standard FEM approximation, a new PU approximation around a crack tip then is constructed as

$$
u^{h}(\mathbf{x})=\sum_{i} N_{i} u_{i}^{\text {loc }}(\mathbf{x})=\sum_{i} N_{i}\left(\sum_{k \in I_{i}} \phi_{k}^{i}(\mathbf{x}) u_{k}\right)
$$

where $N$ is the standard FE shape function and $u_{k}$ is the conventional translational dof. Different from the standard FEM, one individual node appears repeatedly in $n_{i}$ patchwise local approximations in (9),

$$
\underbrace{N_{(i)} \phi_{i}^{(i)} u_{i}}_{\text {patch star node }}, \underbrace{N_{(1)} \phi_{i}^{(1)} u_{i}, N_{(2)} \phi_{i}^{(2)} u_{i}, \cdots, N_{(j), j \neq} \phi_{i}^{(j)} u_{i}, \cdots, N_{\left(n_{i}\right)} \phi_{i}^{\left(n_{i}\right)} u_{i}}_{\text {non patch star nodes }}
$$

where the standard FE shape function is indexed as $N_{(k)}, k=1,2, \ldots, n_{i}$, the bracket of " $(k)$ " implies local numbering of the nodes on patch $i, \phi_{(i)}^{k}$ is the local function constructed on patch $k$ with regard to its $i$-th node, which is different from $\phi_{k}^{i}$ in (9). 
After regrouping the terms in (10) in terms of $u_{i}$, the approximation around the crack tip can be written as

$$
u^{h}(\mathbf{x})=\sum_{i}\left(\sum_{k \in J_{i}} N_{k} \phi_{i}^{k}(\mathbf{x})\right) u_{i}=\sum_{i} N_{i}^{\mathrm{tip}} u_{i}
$$

$N_{i}^{\text {tip }}$ denotes the new shape function of the nodes around the crack tip. The new approximation offers two unique features: (1) no extra dof, all nodal dofs are conventional; (2) the new PU approximation interpolates as $\phi_{i}^{i}\left(\mathbf{x}_{i}\right) \equiv 1$ and $N_{i}^{\mathrm{tip}}\left(\mathbf{x}_{i}\right)=N_{i}\left(\mathbf{x}_{i}\right)$.

\subsection{Crack representation}

In crack analysis, the sub-domain $\Omega^{\text {tip }}$ moves along with its crack tip. The size of $\Omega^{\text {tip }}$ is usually two or three layers of elements (Figure 1). The approximation of $i \mathrm{XFEM}$ is expressed as

$$
u^{h}(\mathbf{x})=\sum_{i \in I} N_{i} u_{i}+\sum_{i \in K} N_{i}\left(\begin{array}{ll}
1 & \tilde{H}_{i}
\end{array}\right)\left(\begin{array}{c}
u_{i} \\
d_{i}
\end{array}\right)+\sum_{i \in J} N_{i}^{\mathrm{tip}} u_{i}
$$

where $I, J, K$, respectively, denote the set of standard FE nodes, tip enrichment nodes, and Heaviside enrichment nodes, $I \cap J \cap K=0$, and $d_{i}$ are the extra dofs due to the Heaviside enrichment $\tilde{H}_{i}=H(\mathbf{x})-H\left(\mathbf{x}_{i}\right)$. The approximation is different from XFEM only in the last term, i.e., the way of tip enrichment, which now does not contain any extra dof.

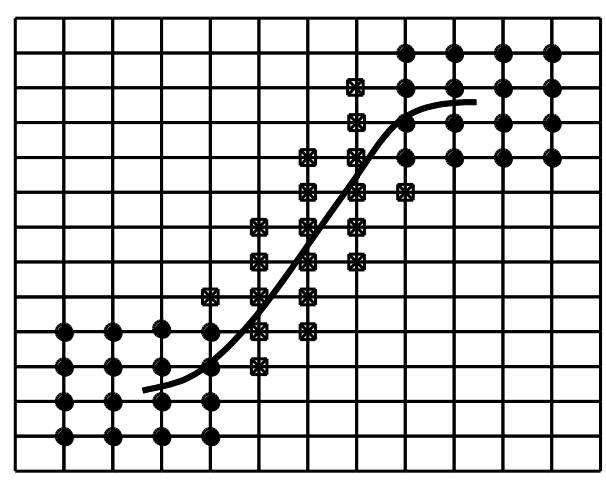

- nodal set $J \quad \otimes$ nodal set $K$

Figure 1. Crack modeling using $i$ XFEM.

\subsection{Blending element treatment}

Due to the loss of the partition of unity property, blending elements can severely affect accuracy and convergence [42]-[44]. For the purpose of blending element treatment in $i$ XFEM, the domain of interest is decomposed in an overlapped manner: $\Omega_{\mathrm{b}}=\Omega_{\text {ntip }} \cap \Omega_{\text {tip }}$, where $\Omega_{\mathrm{b}}$ denotes the overlapping/blending domain, $\Omega_{\text {tip }}$ denotes the tip-enrichment zone, and $\Omega_{\text {ntip }}$ denotes the non-tip enrichment zone composed of both standard and Heaviside enrichment elements.

In $\Omega_{\text {tip }}, i X F E M$ is defined as

$$
u_{\text {tip }}^{h}(\mathbf{x})=\sum_{i \in J} N_{i}^{\mathrm{tip}} u_{i}
$$

which is the same as that in (12). In $\Omega_{\text {ntip }}, i$ XFEM is the same as XFEM 


$$
u_{\mathrm{ntip}}^{h}(\mathbf{x})=\sum_{i \in I+I^{*}} N_{i} u_{i}+\sum_{i \in K+K^{*}} N_{i}\left(\begin{array}{ll}
1 & \tilde{H}_{i}
\end{array}\right)\left(\begin{array}{c}
u_{i} \\
d_{i}
\end{array}\right)
$$

where $I^{*}$ and $K^{*}$ are respectively the standard and the Heaviside enrichment nodes in the overlapping/blending domain $\Omega_{\mathrm{b}}$. Nodal set $I$ and $K$ in (12) are now expanded into the blending domain.

A blending function is defined as the summation of all the standard FE shape functions at the tip enriched nodes except those on the boundary of $\Omega^{\text {tip }}$

$$
R(\mathbf{x})=\sum_{i \in J \backslash J^{*}} N_{i}(\mathbf{x})
$$

where $J^{*}$ denotes the node set along $\partial \Omega_{\text {tip }}, \quad R(\mathbf{x})$ is the so-called ramp function [36], which varies monotonously between 1 and 0 in $\Omega_{\mathrm{b}}$ and expresses as $R$ in the following equations. The global approximation on the whole domain is taken to be a weighted summation of $u_{\text {ntip }}^{h}$ and $u_{\text {tip }}^{h}$,

$$
u^{h}(\mathbf{x})=(1-R) u_{\text {ntip }}^{h}+R u_{\text {tip }}^{h}
$$

$i \mathrm{XFEM}$ is finally expressed as

$$
u^{h}(\mathbf{x})=\sum_{i \in I+I^{*}} \tilde{N}_{i} u_{i}+\sum_{i \in K+K^{*}} \tilde{N}_{i}\left(\begin{array}{ll}
1 & \tilde{H}_{i}
\end{array}\right)\left(\begin{array}{l}
u_{i} \\
d_{i}
\end{array}\right)+\sum_{i \in J} \tilde{N}_{i}^{\mathrm{tip}} u_{i}
$$

where

$$
\begin{gathered}
\tilde{N}_{i}= \begin{cases}(1-R) N_{i} & i \in I+I^{*}, K+K^{*} \\
R N_{i} & i \in J\end{cases} \\
\tilde{N}_{i}^{\mathrm{tip}}=\sum_{k \in J_{i}} \tilde{N}_{k} \phi_{i}^{k}(\mathbf{x})
\end{gathered}
$$

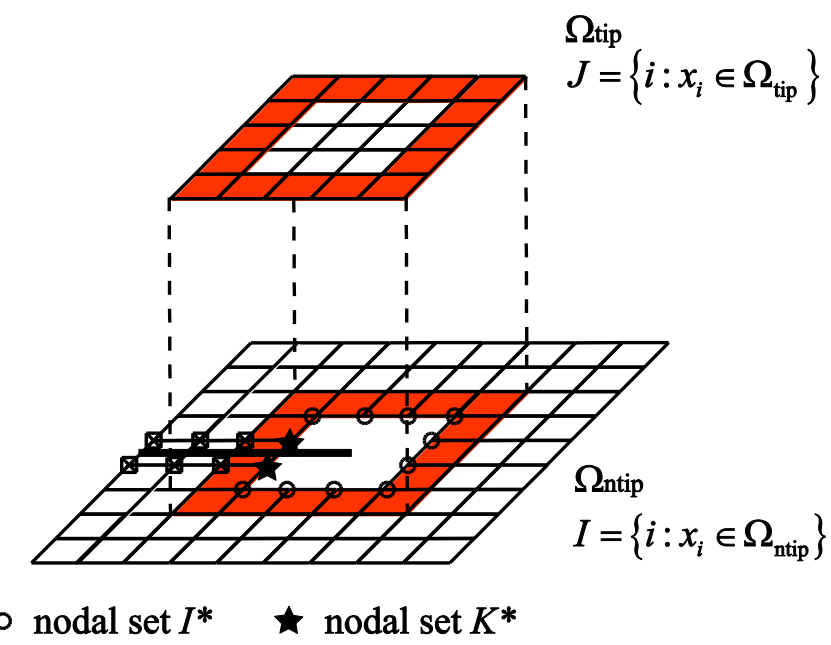

Figure 2. Notations in blending element treatment.

\section{Discrete equations}

We consider a problem of small displacement, linear elastic dynamics on domain $\Omega$ with boundary $\Gamma$, where $\Gamma_{\mathrm{t}}$ is the prescribed traction boundary and $\Gamma_{u}$ is the 
prescribed displacement boundary. The strong form of the problem is as follows

$$
\begin{gathered}
\operatorname{div}(\boldsymbol{\sigma})+\rho_{0} \mathbf{b}=\rho_{0} \ddot{\mathbf{u}} \text { in } \Omega \\
\mathbf{u}=\overline{\mathbf{u}} \text { on } \Gamma_{u} \\
\boldsymbol{\sigma} \cdot \mathbf{n}=\overline{\mathbf{t}} \text { on } \Gamma_{\mathrm{t}} \\
\mathbf{u}(\mathbf{x}, 0)=\mathbf{u}_{0}(\mathbf{x}), \dot{\mathbf{u}}(\mathbf{x}, 0)=\dot{\mathbf{u}}_{0}(\mathbf{x}) \text { in } \Omega \\
\boldsymbol{\varepsilon}=\nabla_{s} \mathbf{u}, \quad \boldsymbol{\sigma}=\mathbf{C}: \boldsymbol{\varepsilon}
\end{gathered}
$$

where $\sigma$ is the Cauchy stress tensor for a displacement field $\mathbf{u}, \mathbf{b}$ is the body force vector, $\rho_{0}$ is the material density, $\mathbf{n}$ is the unit outward normal vector to the traction boundary $\Gamma_{\mathrm{t}}, \nabla_{s}$ is the symmetric gradient operator, $\mathbf{C}$ is the elastic module tensor. The superposed bar denotes the prescribed values at the boundaries. The weak form of the momentum equation in the current configuration is then given by

$$
\int_{\Omega} \rho \delta \mathbf{u}: \ddot{\mathbf{u}} \mathrm{d} \Omega+\int_{\Omega} \nabla_{s} \delta \mathbf{u}: \boldsymbol{\sigma} \mathrm{d} \Omega-\int_{\Omega} \delta \mathbf{u} \cdot \mathbf{b} \mathrm{d} \Omega-\int_{\Gamma_{t}} \delta \mathbf{u} \cdot \overline{\mathbf{t}} \mathrm{d} \Gamma=0
$$

Because the tip enriched shape function is related to the tip position $\mathbf{x}_{\text {tip }}(t)$ that is the function of time (which is the same as XFEM),

$$
\phi_{i}^{k}(\mathbf{x}, t)=\phi_{i}^{k}\left(\mathbf{x}-\mathbf{x}_{\text {tip }}(t)\right)
$$

the time derivative of the tip enriched shape function can be derived as

$$
\begin{gathered}
\dot{\phi}_{i}^{k}(\mathbf{x}, t)=-\dot{\mathbf{x}}_{\text {tip }}(t) \nabla \phi_{i}^{k} \\
\ddot{\phi}_{i}^{k}(\mathbf{x}, t)=-\ddot{\mathbf{x}}_{\text {tip }}(t) \nabla \phi_{i}^{k}+\left\|\dot{\mathbf{x}}_{\text {tip }}(t)\right\|^{2} \nabla^{2} \phi_{i}^{k}
\end{gathered}
$$

where $\dot{\mathbf{x}}_{\text {tip }}(t)$ and $\ddot{\mathbf{x}}_{\text {tip }}(t)$ are respectively the velocity and the acceleration of the crack tip of interest. These time derivative terms, according to our tests, have no much effect on accuracy. They are provided here only for theoretical completeness - they can simply neglected.

Accordingly, we have

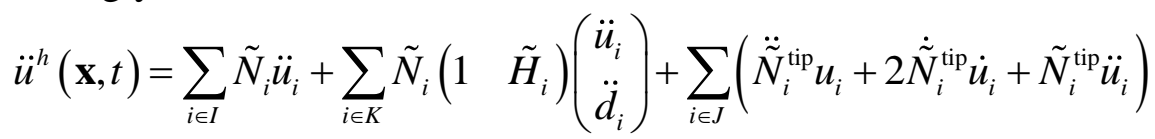

where

$$
\ddot{\tilde{N}}_{i}^{\text {tip }}=\sum_{k \in J_{i}} \tilde{N}_{k}(\mathbf{x}) \ddot{\phi}_{i}^{k}(\mathbf{x}, t) ; \quad \dot{\tilde{N}}_{i}^{\text {tip }}=\sum_{k \in J_{i}} \tilde{N}_{k}(\mathbf{x}) \dot{\phi}_{i}^{k}(\mathbf{x}, t)
$$

The configuration $\mathbf{x}$ is assumed to be time invariant under the assumption of infinitesimal deformation, i.e. $\mathbf{x} \equiv \mathbf{X}$.

The Galerkin-form of discretization of Eq.(25) leads to the following system of linear equations

$$
\mathbf{M U ̈}+\mathbf{K U}=\mathbf{F}^{\mathrm{ext}}-\mathbf{F} \quad t \in[0, \mathrm{~T}]
$$

where

$$
\ddot{\mathbf{U}}=\left\{\begin{array}{l}
\ddot{\mathbf{u}}_{I} \\
\ddot{\mathbf{u}}_{J} \\
\ddot{\mathbf{u}}_{K} \\
\ddot{\mathbf{d}}
\end{array}\right\}, \dot{\mathbf{U}}=\left\{\begin{array}{l}
\dot{\mathbf{u}}_{I} \\
\dot{\mathbf{u}}_{J} \\
\dot{\mathbf{u}}_{K} \\
\dot{\mathbf{d}}
\end{array}\right\}, \mathbf{F}=\left\{\begin{array}{l}
\mathbf{0} \\
\mathbf{F}_{J} \\
\mathbf{0} \\
\mathbf{0}
\end{array}\right\}
$$


F is the time derivatives of $\tilde{N}_{i}^{\text {tip }}$,

$$
\mathbf{F}_{J}=\left(\int_{\Omega^{\mathrm{tip}}}\left[\tilde{\mathbf{N}}^{\mathrm{tip}}\right]^{\mathrm{T}} \ddot{\mathbf{N}}^{\mathrm{tip}} \mathrm{d} \Omega\right) \mathbf{u}_{J}+2\left(\int_{\Omega^{\mathrm{tip}}}\left[\tilde{\mathbf{N}}^{\mathrm{tip}}\right]^{\mathrm{T}} \dot{\tilde{\mathbf{N}}}^{\mathrm{tip}} \mathrm{d} \Omega\right) \dot{\mathbf{u}}_{J}
$$

which vanishes when the crack is stationary,

$$
\begin{gathered}
\mathbf{M}=\int_{\Omega} \rho \mathbf{N}^{\mathrm{T}} \mathbf{N} \mathrm{d} \Omega \\
\mathbf{K}=\int_{\Omega} \mathbf{B}^{\mathrm{T}} \mathbf{D B} \mathrm{d} \Omega \\
\mathbf{F}^{\mathrm{ext}}=\int_{\Omega} \mathbf{N}^{\mathrm{T}} \mathbf{b} \mathrm{d} \Omega+\int_{\Gamma_{t}} \mathbf{N}^{\mathrm{T}} \overline{\mathbf{t}} \mathrm{d} \Gamma \\
\mathbf{N}=\left[\mathbf{N}_{1}, \mathbf{N}_{2}, \ldots, \mathbf{N}_{N}\right], \mathbf{N}_{i}=\left[\begin{array}{cc}
\phi_{i} & 0 \\
0 & \phi_{i}
\end{array}\right] \\
\mathbf{B}=\nabla_{s} \mathbf{N}, \nabla_{s}\left(\mathbf{N}_{i}\right)=\left[\begin{array}{cc}
\phi_{i, x} & 0 \\
0 & \phi_{i, y} \\
\phi_{i, y} & \phi_{i, x}
\end{array}\right] \\
\phi_{i}= \begin{cases}\tilde{N}_{i} & i \in I+I^{*} \\
\tilde{N}_{i}^{\mathrm{tip}} & i \in J \\
\tilde{N}_{i} \tilde{H}_{i} & i \in K+K^{*}\end{cases}
\end{gathered}
$$

D is the elasticity matrix.

\section{Time integration scheme}

The Newmark method [23] is chosen as the implicit time integrator. The updating equations are given by

$$
\begin{aligned}
& \dot{\mathbf{U}}_{n+1}=\dot{\mathbf{U}}_{n}+\Delta t(1-\beta) \ddot{\mathbf{U}}_{n}+\Delta t \beta \ddot{\mathbf{U}}_{n+1} \\
& \mathbf{U}_{n+1}=\mathbf{U}_{n}+\Delta t \dot{\mathbf{U}}_{n}+\Delta t^{2}\left(\frac{1}{2}-\alpha\right) \ddot{\mathbf{U}}_{n}+\Delta t^{2} \alpha \ddot{\mathbf{U}}_{n+1} \\
& \tilde{\mathbf{K}} \mathbf{U}_{n+1}=\Delta \tilde{\mathbf{F}} \\
& \tilde{\mathbf{K}}=\mathbf{K}+\frac{1}{\alpha \Delta t^{2}} \mathbf{M} \\
& \Delta \tilde{\mathbf{F}}=\Delta \mathbf{F}+\left[\left(\frac{1}{2 \alpha}-1\right) \ddot{\mathbf{U}}_{n}+\frac{1}{\alpha \Delta t} \dot{\mathbf{U}}_{n}\right] \mathbf{M}
\end{aligned}
$$

where $\mathbf{U}_{n}, \dot{\mathbf{U}}_{n}$ and $\ddot{\mathbf{U}}_{n}$ are the global vectors of nodal displacements, nodal velocities and nodal accelerations, respectively. $\Delta t$ is the time increment at the present time step and the Newmark's parameters are chosen to be $\beta=1 / 4$ and $\alpha=1 / 2$ to fulfill the unconditionally stable criterion.

For an explicit time integrator, the central difference method is chosen, which corresponds to $\beta=1 / 2$ and $\alpha=0$. The equations discretized in space and time are as follow

$$
\mathbf{U}_{n+1}=\mathbf{U}_{n}+\Delta t \dot{\mathbf{U}}_{n}+\frac{1}{2} \Delta t^{2} \ddot{\mathbf{U}}_{n}
$$




$$
\begin{gathered}
\mathbf{M} \ddot{\mathbf{U}}_{n+1}=\mathbf{F}_{n+1}-\mathbf{K} \mathbf{U}_{n+1} \\
\dot{\mathbf{U}}_{n+1}=\dot{\mathbf{U}}_{n}+\frac{1}{2} \Delta t\left(\ddot{\mathbf{U}}_{n}+\ddot{\mathbf{U}}_{n+1}\right)
\end{gathered}
$$

The scheme is conditionally stable, the time step must be chosen in accordance with the Courant-Friedrichs-Lewy condition,

$$
\Delta t \leq \Delta t_{c}=\frac{2}{\omega_{\max }}
$$

where $\omega_{\max }$ is the maximum frequency determined by the following equation:

$$
\operatorname{det}\left(\mathbf{K}-\omega^{2} \mathbf{M}\right)=0
$$

when the mass matrix $\mathbf{M}$ is lumped, the solution of the problem does not need to solve the linear system.

\section{Mass lumping and dof dynamics}

\subsection{Mass lumping}

In 2006, Menouillard et al. [23] introduced the first mass matrix lumping technique for Heaviside enrichment on the cut element. The method is based on the exact kinetic energy representation of the basic motions,

$$
m=\frac{1}{N_{\text {node }}} \int_{\Omega^{e}} \rho H^{2} \mathrm{~d} \Omega=\frac{m_{e}}{N_{\text {node }}}
$$

where $e$ is the element cut by crack, $N_{\text {node }}$ is the node number of element $e, m_{e}$ is the mass of the element. In 2008, Rozycki et al. [48] proposed a mass lumping technique for the constant strain elements containing holes and free boundaries.

In 2009, Elguedj and his co-workers [32] followed the idea of Menouillard et al. [23] and presented a general mass lumping technique for an arbitrary enrichment function $\varphi$,

$$
m=\frac{1}{\sum_{i=1}^{N_{\text {node }}} \varphi^{2}\left(\mathbf{x}_{i}\right)} \int_{\Omega^{e}} \rho \varphi^{2} \mathrm{~d} \Omega
$$

This mass lumping technique is currently used for both the Heaviside and the tip enriched elements in XFEM.

In $i$ XFEM, as there is no extra dof in the crack tip enrichment domain $\Omega^{\mathrm{tip}}$, the standard mass lumping process of FEM can be followed. On the Heaviside enrichment element, iXFEM uses the same mass lumping scheme of XFEM, i.e., Eq.(46).

\subsection{Dof dynamics}

Besides the above mass lumping, the most daunting issue in the development history of XFEM is the conservative energy transfer between time steps. As the crack grows, a tip enrichment element in this step may become a Heaviside enrichment element in the next. The difference in enrichment between the time steps leads to the change of extra dofs, as well as the generalized nodal velocities and accelerations. If simply discarding the tip enrichment in the previous time step and switching to the Heaviside enrichment, energy cannot be properly transferred to the next time step and there happens a sudden loss of accuracy once if the crack propagates from one element into another.

The problem is not solved until 2005 by Réthoré et al. [28]. The ideas are to 
continuously add an additional set of extra dofs for each new tip location; while keeping those extra dofs, Heaviside enrichment extra dofs are further appended if the current element becomes a cut element; No any extra dofs are discarded during the simulation. This is shown in Figure 3. It can be verified that this approach can assure the conservative energy transfer between the time steps.

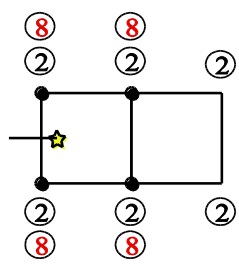

step 1

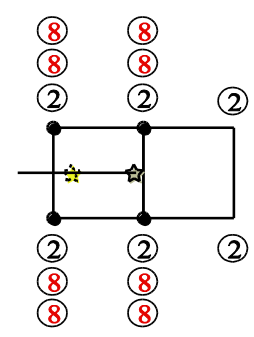

step 2

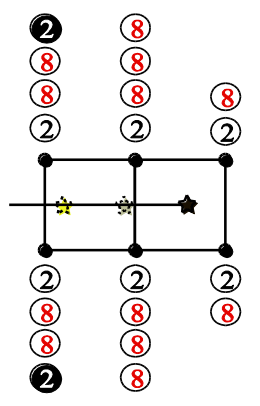

step 3

(2) Dofs of standard displacement (8) Dofs of crack tip enrichment

2 Dofs of Heaviside enrichment - Crack tip enrichment node

Figure 3. Nodal dofs for implicit time integration in XFEM. "2" and " 8 " are the numbers of dof corresponding to the standard or Heaviside shape function, and the tip shape function.

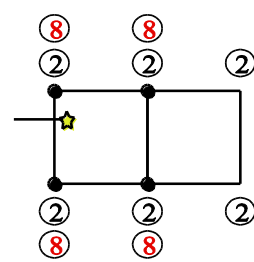

(a) Initial problem

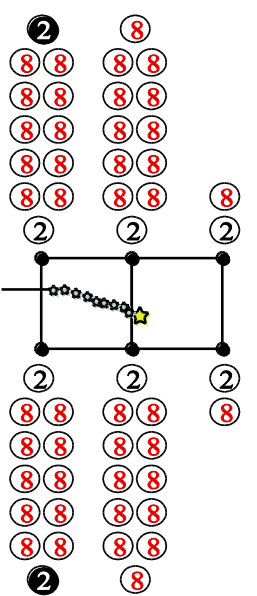

(b) Crack propagate 10 steps into a new element

Figure 4. Nodal dofs for explicit time integration in XFEM.

The above nodal enrichment, which is theoretically strict in terms of energy transfer on tip extra dofs, could be problematic for two reasons. One is the bad conditioning in an implicit time integrator. Unless we enforce a crack to grow one element size each step, the successive crack growth within element would introduce an increased number of nodal extra dofs and hence elevated bad-conditioning. The other is the excessive number of nodal extra dofs in an explicit time integrator. A stable explicit time stepping requires a crack propagate at least 10 steps before it moves out of an element (growth steps per element: $\frac{2}{c \dot{a}} \sqrt{E /\left(\rho(1-v)^{2}\right)}$ ). In the case of a square element, the number of steps is 11.3 and more than 352 extra dofs would be introduced to one element (Figure 4). The dynamic nature of the dofs system complicates very much the implementation of XFEM in dynamic crack growth simulation. 
In contrast, since there is no extra dof at crack tip in $i$ XFEM, the above nodal enrichment procedure is avoided completely. If the crack tip propagates within the same element, e.g., Figure 5 step 2, the tip enriched nodes contain just the translational dofs (of course the tip enriched shape function should account for the new tip position). If the crack propagates into a new element, as shown in Figure 5 step 3, $i$ XFEM constructs a new tip enrichment subdomain. For those old tip enriched nodes that now do not longer belong to the new tip enrichment subdomain, Heaviside dofs, i.e., the $H$ dofs, are added and the shape function is also changed accordingly. Such a procedure is exactly the same as that in a static crack analysis.

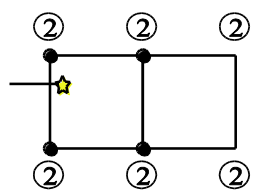

step 1

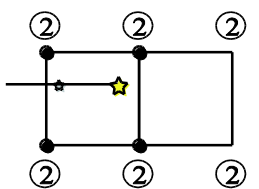

step 2

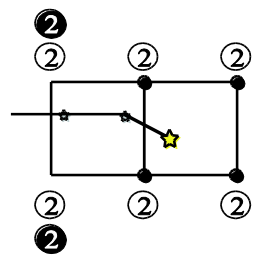

step 3

(2) Dofs of standard displacement

- Crack tip enrichment node

Figure 5. Nodal dofs in $i$ XFEM during crack propagation. " 2 " is the number of dofs of the standard or the Heaviside function.

\section{Crack opening displacement (COD) compatibility issue}

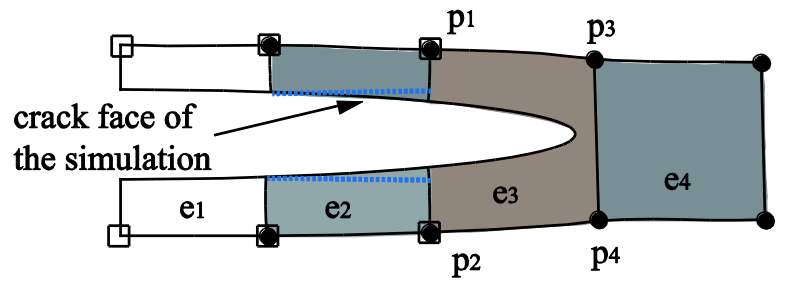

(a) time step $n$

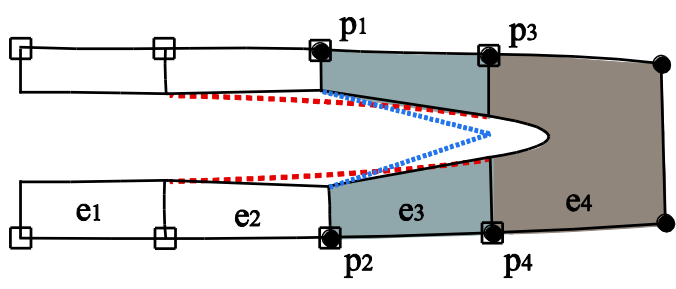

blending element

tip element

- tip enrichment

$\square \quad$ Heaviside enrichment

...- crack face before crack propagation

....... crack face of Heaviside enrichment in cut blending element

(b) time step $\mathrm{n}+1$

Figure 6. COD imcompatility issue.

In a continuous blending element, the ramp function implements a smooth transition of displacement approximation from $u_{\text {ntip }}^{h}$ to $u_{\text {tip }}^{h}$. Careful investigation, however, found that this blending element treatment does not necessarily assure COD compatibility along the crack surface in the cracked blending element. This can be clearly seen by plotting the CODs respectively using the Heaviside approximation and the blending approximation in the blending element (Figure 7(a)).

This COD incompatibility issue happens for two reasons: it is first introduced 
when new Heaviside dofs ( $H$ dofs) are added to, for example, $p_{3}$ and $p_{4}$ at step $n+1$ with incorrect "zero" initial values (Figure 6). And then the error is magnified due to the change of the approximation function on the cracked blending element from the blending form to the Heaviside form as the crack propagates into next element and the blending element becomes a purely cut element. For example, when the tip element $e_{3}$ at time step $n$ becomes the blending element at step $n+1$, the new $H$ dofs a are added to nodes $p_{3}$ and $p_{4}$. The initial values of $\mathbf{a}, \dot{\mathbf{a}}, \ddot{\mathbf{a}}$ are zero only when the crack tip is passing exactly $\overline{p_{3} p_{4}}$----once the crack crossed the edge, the initial values of $\mathbf{a}, \dot{\mathbf{a}}, \ddot{\mathbf{a}}$ must not be zero. This introduces the first errors. Then once the cracked blending element becomes a purely cut element and the Heaviside shape function on the element is used instead of the blending shape function, the crack opening cannot be reproduced accurately and the error is magnified. This issue mainly affects dynamic crack growth simulation.

The COD incompatibility issue can be solved easily by enforcing $u^{\mathrm{b}}(x)=u^{\mathrm{H}}(x)$ in a weak sense:

$$
\int_{\Omega^{\mathrm{H}}} N_{i}\left(u^{\mathrm{b}}(x)-u^{\mathrm{H}}(x)\right) \mathrm{d} \Omega=0
$$

where $N_{i}$ is the standard FEM shape function, $u^{\mathrm{b}}(x)$ and $u^{\mathrm{H}}(x)$ are, respectively, the blending and the Heaviside approximation, $\Omega^{\mathrm{H}}$, in logic, should contain the three blending elements containing $p_{3}$ and $p_{4}$ in this $2 \mathrm{D}$ case, but due to the shifted form of Heaviside enrichment, $u^{\mathrm{b}}(x)=u^{\mathrm{H}}(x)$ is satisfied node-wise on the continuous blending elements and hence we mainly integrate the constraint on the cracked blending element.

Take time step $n+1$ as the configuration of interest, the blending approximation on the cracked blending element $e_{3}$ is

$$
u^{\mathrm{b}}(x)=(1-R(x)) u^{\mathrm{H}}(x)+R(x) u^{\mathrm{tip}}(x)
$$

where $R(x)$ is the ramp function. Substituting (49) into (48), we get the following system of linear equations with regard to the newly added $H$ dofs $d_{3 x}, d_{3 y}, d_{4 x}, d_{4 y}$

$$
\mathbf{C d}=\Delta \mathbf{U}
$$

where

$$
\begin{gathered}
\mathbf{C}=\int_{e_{3}}\left[\begin{array}{c}
R \mathbf{N}_{3} \\
R \mathbf{N}_{4}
\end{array}\right]_{4 \times 2}\left[\left(H-H_{3}\right) \mathbf{N}_{3} \quad\left(H-H_{4}\right) \mathbf{N}_{4}\right]_{2 \times 4} \mathrm{~d} \Omega \\
\Delta \mathbf{U}=\int_{e_{3}}\left[\begin{array}{c}
R \mathbf{N}_{3} \\
R \mathbf{N}_{4}
\end{array}\right]_{4 \times 2}\left(\begin{array}{c}
\Delta u_{x} \\
\Delta u_{y}
\end{array}\right) \mathrm{d} \Omega \\
\mathbf{N}_{i}=\left[\begin{array}{cc}
N_{i} & 0 \\
0 & N_{i}
\end{array}\right], \quad \Delta u=u^{\text {tip }}-u^{\text {fem }}-\sum_{i=1,2}\left(H-H_{i}\right) N_{i} d_{i}
\end{gathered}
$$

$\mathbf{d}=\left[d_{3 x}, d_{3 y}, d_{4 x}, d_{4 y}\right]^{\mathrm{T}}$ are the degrees of freedom newly added to nodes $p_{3}$ and $p_{4}$. $u^{\text {tip }}=\sum_{i} N_{i}^{\text {tip }} u_{i}$ is the approximation using the crack tip enrichment, $u^{\mathrm{fem}}=\sum_{i} N_{i} u_{i}$ is the approximation of standard FEM. By solving the $4 \times 4$ linear system, we can obtain the corrected $H$ dofs at $p_{3}$ and $p_{4}$.

Similarly, the time derivatives of Equ.(48) give the constraints for $\dot{u}(x)$ and $\ddot{u}(x)$ to be satisfied. In the same manner, we obtain the corrected $\dot{d}_{3 x}, \dot{d}_{3 y}, \dot{d}_{4 x}, \dot{d}_{4 y}$ and $\ddot{d}_{3 x}, \ddot{d}_{3 y}, \ddot{d}_{4 x}, \ddot{d}_{4 y}$ at $p_{3}$ and $p_{4}$. With these modifications, the COD incompatibility issue 
is solved as shown in Figure 7(b)-(d). The correction can be done posterior after each time step and hence it is easy to implement.

We remark that the above COD incompatibility issue should also apply to XFEM and the similar correction may be suggested applying to XFEM to find proper initial values of the newly added $H$ dofs.
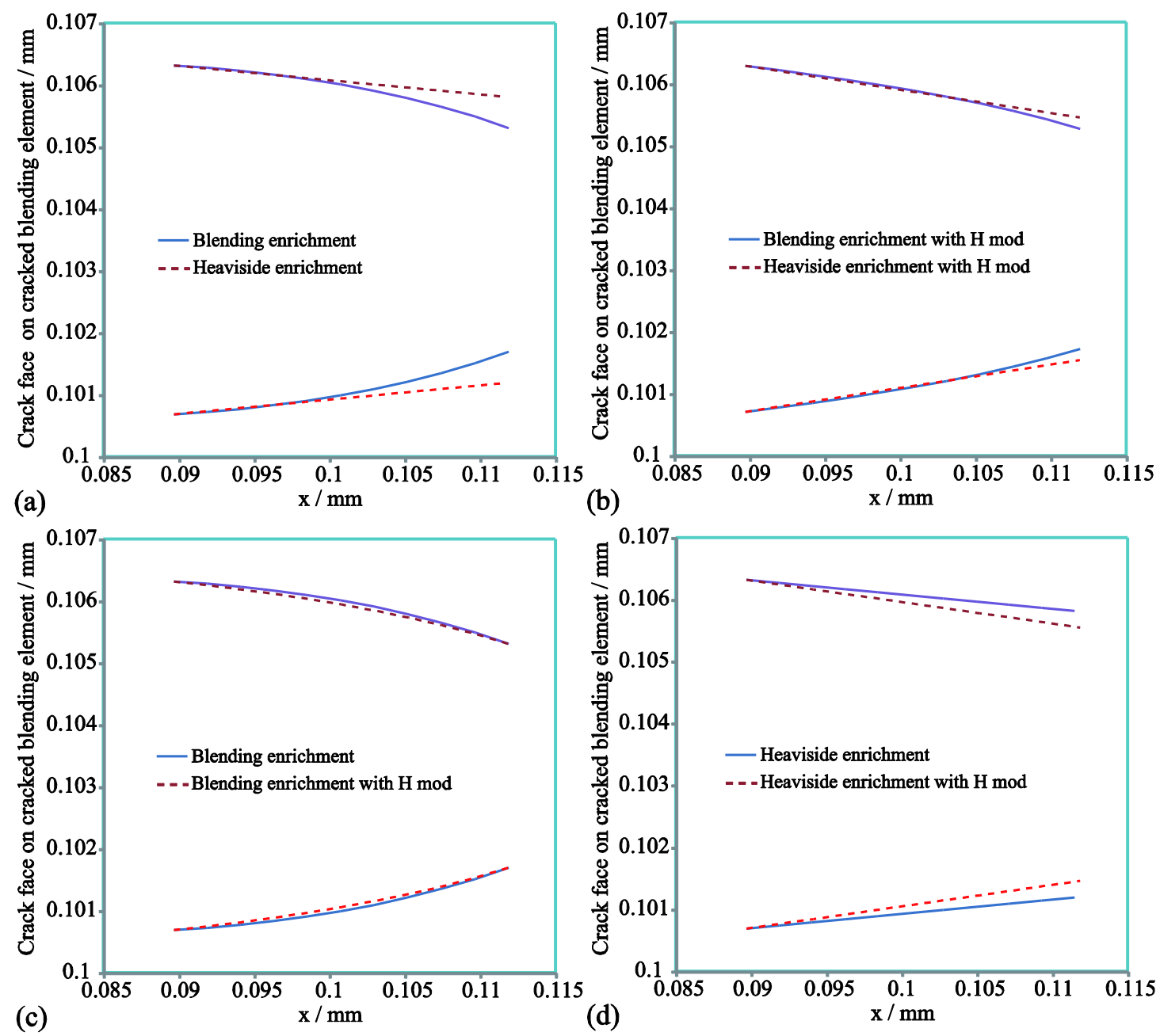

Figure 7. CODs on cracked blending element. (a) before the $H$ modification, (b) after the $H$ modification, (c) Blending enrichment before and after the $H$ modification, (d) Heaviside enrichment before and after the $H$ modification.

\section{Numerical examples}

Quadrature rules are set as follows, which apply to the most of examples:

- The element containing crack tip (if the case) is triangulated by Delaunay triangulation algorithm and each sub-triangle is integrated by 13-point quadrature;

- If the crack tip is located on an element edge, the element ahead is also triangulated and each sub-triangle is integrated by 13 -point quadrature;

- If the crack tip is located at a node, the surrounding $2 \times 2$ elements are triangulated and each sub-triangle is integrated by 13-point quadrature;

- On the split/cut (Heaviside enriched) element, each split part is triangulated and each sub-triangle is integrated by 9 -point quadrature; 
- The intact enriched elements and the intact blending elements are integrated by $4 \times 4$ Gauss quadrature.

- Non-enriched elements are integrated by $2 \times 2$ Gauss quadrature.

\subsection{Effects of mass lumping on critical time step size}

In 2006, Menouillard et al. [23] proposed a mass lumping technique for Heaviside enriched elements and suggested the critical time step in explicit transient analysis to be about $2 / 3 \Delta t_{\text {fem }}^{\text {lump }}$ where $\Delta t_{\text {fem }}^{\text {lump }}$ is the critical time step size of the standard FEM. In 2009, Elguedj et al. [32] introduced a general mass lumping technique for tip enriched elements and the critical time step size of the quadrilateral tip enriched element is found to be $2 / 3 \Delta t_{\text {fem }}^{\text {lump }}$ for the stationary crack and $1 / 2 \Delta t_{\text {fem }}^{\text {lump }}$ for the moving crack. Then, Elguedj et al. [31] suggested using $1 / 2 \Delta t_{\text {fem }}^{\text {lump }}$ as the time step size in explicit transient analysis when both the Heaviside and the tip enrichments present.

Since the tip enrichment in XFEM tends to shorten the critical time step size, the effect of the crack tip enrichment in $i$ XFEM on the critical time step size is examined. As the tip enrichment strategy in $i$ XFEM is identical no matter the crack is stationary or moving, only the stationary case is tested.

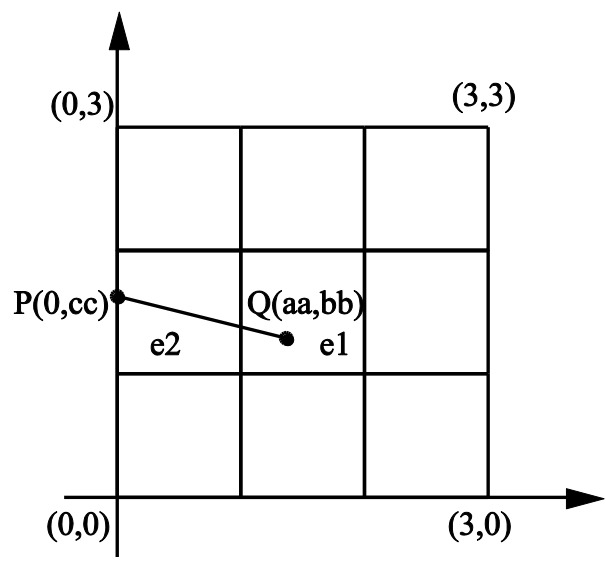

Figure 8. Effects of mass lumping on critical time step size.

Consider the plate with an edge crack shown in Figure 8. The dimension of the plate is $3 \mathrm{~m} \times 3 \mathrm{~m}$, density is $\rho=1 \mathrm{~kg} / \mathrm{m}^{3}$, Young's modulus is $E=1000 \mathrm{~N} / \mathrm{m}^{2}$, and Poisson's ratio $v=0.3$. A $3 \times 3$ uniform square mesh is used for tests. The left endpoint of the crack is located at the middle of the left-handed boundary and the other endpoint randomly walks in the center element $e 1,[1,2] \times[1,2]$, to represent arbitrary layouts. All nodes on the mesh are tip enriched. The critical time step size is obtained with the consistent mass matrix and the lumped mass matrix, and is normalized by the critical time step size of the uncut element.

Figure 9 shows the plots of the critical time step size versus the tip position. Table 1 gives the minimum critical time step size among all the tip positions. The values are normalized by the critical time step of the standard FEM. Eq.(44) is used to estimate the critical time step size, $\Delta t / \Delta t_{\text {fem }}^{\text {lump }}$. When the whole of domain is tip enriched, the stiffness matrix of XFEM is ill-conditioned, $\omega_{\max }$ of the tip element " $e_{1}$ " is used.

Critical time step sizes are listed and compared in Table 1 and Figure 9. For the lumped mass, the minimum value of the critical time step size of $i$ XFEM is $0.74 \Delta t_{\text {fem }}^{\text {lump }}$ while that of XFEM is $0.61 \Delta t_{\text {fem }}^{\text {lump }}$. For the consistent mass, XFEM shows the "null time 
step size", which is $0.0178 \sim 0.1258 \Delta t_{\text {fem }}^{\text {lump }}$ in our test (see Figure 9(a)). Under the exactly same test condition, $i$ XFEM leads to much favorable time step size: $0.4838 \sim 0.6785 \Delta t_{\text {fem }}^{\text {lump }}$. It is hence concluded that $i$ XFEM shows a more favorable critical step size than XFEM in both the cases of consistent and lumped mass and $i$ XFEM avoids the "null time step size" issue.

In the numerical tests hereinafter, we use the following critical time step size,

$$
\Delta t_{c}^{i \mathrm{XFEM}}=\frac{2}{3} \Delta t_{\mathrm{fem}}^{\mathrm{lump}}
$$

Table 1. Effects of mass lumping on critical time step size.

\begin{tabular}{lcccc}
\hline & min value & $\Delta t / \Delta t_{\text {fem }}^{\text {lump }}$ & max value & $\Delta t / \Delta t_{\text {fem }}^{\text {lump }}$ \\
\hline & consistent & lumped & consistent & lumped \\
XFEM & 0.0178 & 0.6164 & 0.1258 & 0.6785 \\
$i$ XFEM & 0.4838 & 0.7432 & 0.6125 & 0.8496 \\
\hline
\end{tabular}

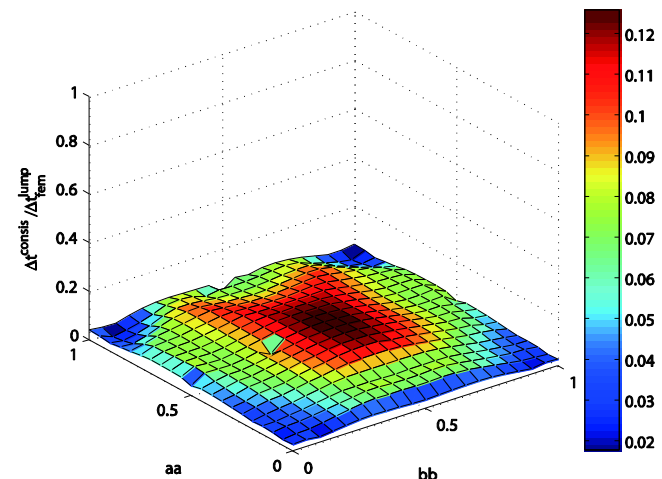

(a) XFEM - consistent mass matrix

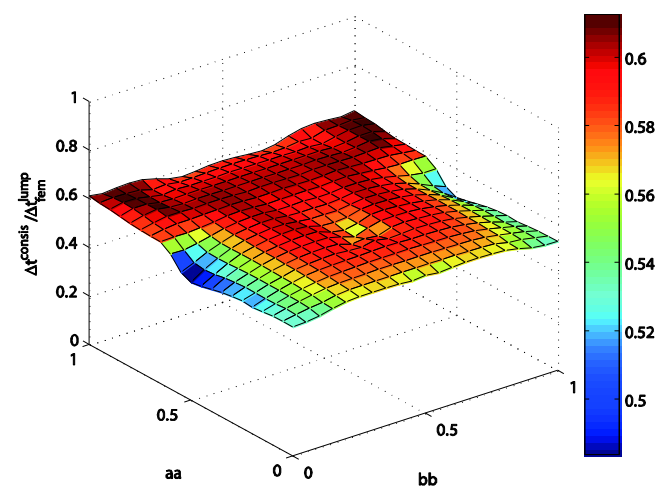

(c) $i$ XFEM - consistent mass matrix

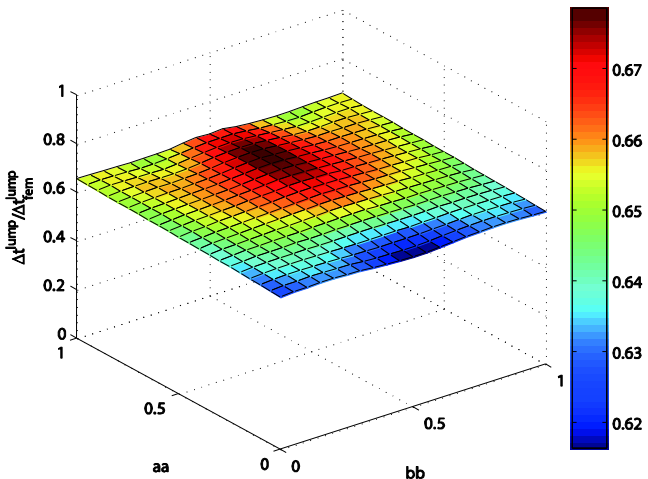

(b) XFEM - lumped mass matrix

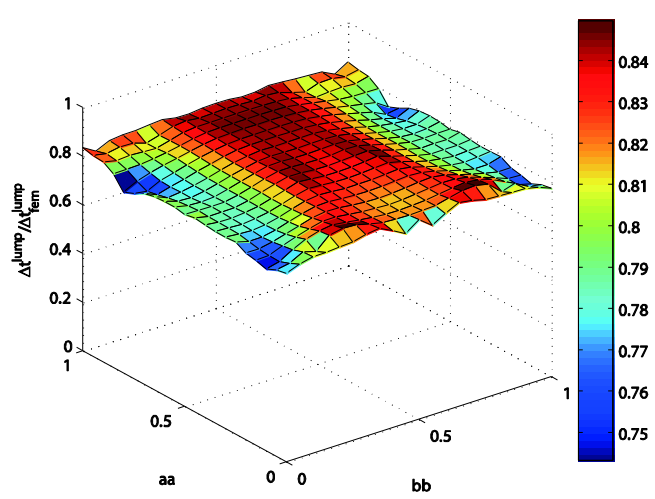

(d) $i$ XFEM - lumped mass matrix

Figure 9. Normalized critical time step size as a function of the crack tip position. (a)

XFEM with consistent mass matrix; (b) XFEM with lumped mass matrix; (c) $i$ XFEM with consistent mass matrix; (d) iXFEM with lumped mass matrix.

\subsection{Mixed mode semi-infinite stationary crack}

The geometry of the problem is $L=4 \mathrm{~m}, H=6 \mathrm{~m}$, the length of the initial crack $a=1$ $\mathrm{m}$, as illustrated in Figure 10. The material properties are: $E=200 \mathrm{GPa}, v=0.25, \rho=$ $7833 \mathrm{~kg} / \mathrm{m}^{3}$, and the prescribed velocity $V_{0}=16.5 \mathrm{~m} / \mathrm{s}^{-1}$. The crack is stationary. The problem is popular for benchmarking the accuracy of dynamic stress intensity factor and has been discussed in literature [25][28][32][34]. The analytical solution of 
dynamic stress intensity factor is given by Lee and Freund [47]. Considering the finite size of the numerical model, the analytical solution is valid only before the reflected stress wave reaches the crack tip, i.e., $t \leq 3 t_{c}=3 a / c_{d}=542 \mu \mathrm{s}$.

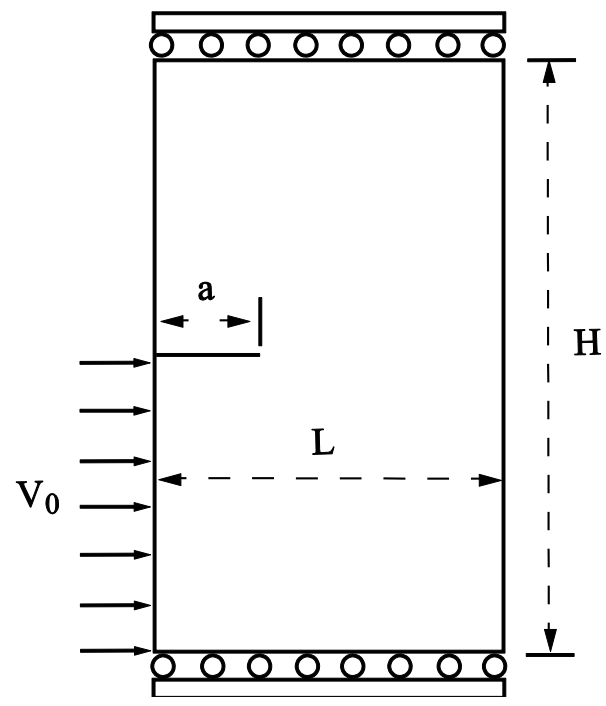

Figure 10. Geometry and loading for mixed mode semi-infinite crack.

A mesh of $39 \times 59$ elements is considered. Time step size is chosen to be $\Delta t=\Delta t_{\mathrm{fem}}^{\text {lump }} / 2=9 \mu \mathrm{s}$. $i \mathrm{XFEM}$ is compared with XFEM for both implicit and explicit time integrators in Figure 11. The value of dynamic stress intensity factor is normalized as $\bar{K}^{\mathrm{dyn}}(t)=K^{\mathrm{dyn}}(t) /\left(-E V_{0} \sqrt{a} /\left(2 c_{d}\left(1-v^{2}\right) \sqrt{\pi}\right)\right)$. The size of enrichment domain, the $J$-domain layout, and the integration scheme are all kept the same for the two methods. It can be seen that $i$ XFEM offers the almost same accuracy as XFEM with consistent mass for implicit time integration and it shows improvement over XFEM with lumped mass for explicit time integration.

In order to circumvent the issue of extra dofs at tip enriched nodes, XFEM is also implemted by the tip enrichment containing only one special function term. In Figure $12, i \mathrm{XFEM}$ is also compared with the XFEM with enrichment function $\sqrt{r} \sin (\theta / 2)$ from reference [25] and the XFEM with enrichment function $r^{2} \sin (\theta / 2)$ from reference [32] for explicit time integration.
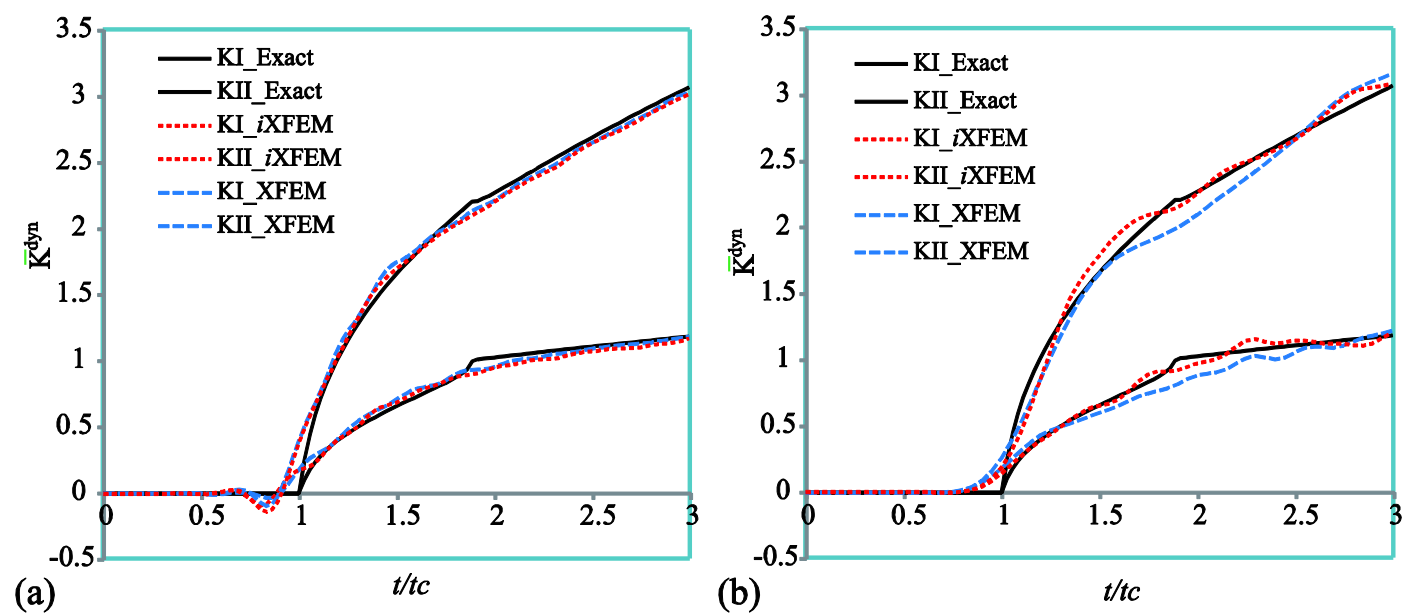

(b) 
Figure 11. Accuracy assessment of dynamic SIF for a stationary mixed mode semiinfinite crack. (a) implicit with consistent mass (b) explicit with lumped mass.

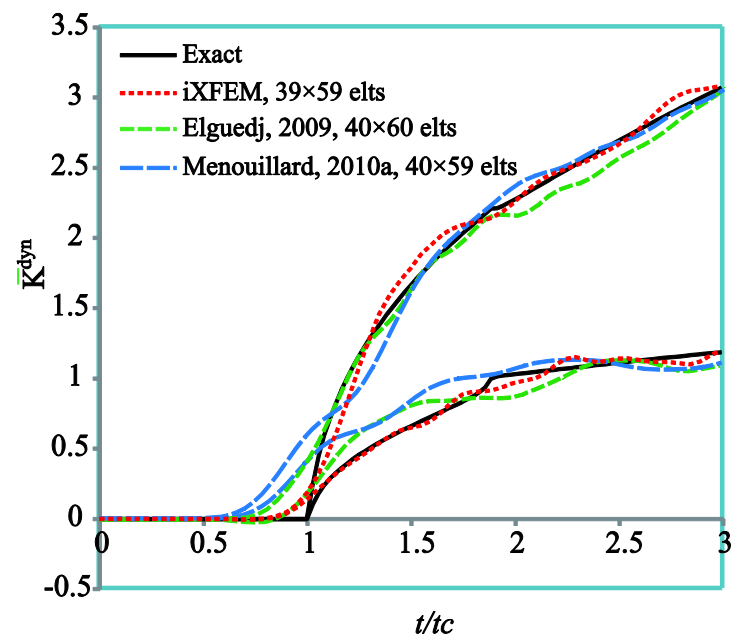

Figure 12. Normalized dynamic stress intensity factors vensus time for explicit time intergrator.

\subsection{Double cantilever beam problem: assessment on conservative energy transfer}

The example has been investigated by Réthoré [28][53] and Combescure [35] to evaluate energy conservative passing between time steps in XFEM. The same benchmark is repeated below with $i$ XFEM to provide an insight into the energy consistency property of the new method.

The geometry of the problem is shown in Figure 13, where $c=0.01 \mathrm{~m}, b=0.05$ $\mathrm{m}$, and the crack length $a=0.02 \mathrm{~m}$. The material parameters are: $E=186 \mathrm{GPa}, v=0.3$, $\rho=8000 \mathrm{~kg} / \mathrm{m}^{3}$ and the fracture toughness $K_{\mathrm{IC}}=110 \mathrm{MPa} \mathrm{m}{ }^{1 / 2}$. A prescribed vertical displacement is applied at the end of the specimen linearly elevating from 0 to $u_{d}=$ $0.0025 \mathrm{~m}$ within $0.1 \mathrm{~ms}$. The maximum hoop stress criterion is employed and the velocity of crack tip is calculated by Equ.(68).

A uniform mesh of $9 \times 49$ elements is employed. The dynamic problem is solved by the implicit time intergration with both consistent and lumped mass matrices. Time step is chosen as $\Delta t=0.5 \mu \mathrm{s}$. The size of $\Omega^{\text {tip }}$ is taken to be $1.0 \mathrm{~h}$ and the size of the $J$ domain to be $2.0 h$, where $h$ is the mesh size. The simulation was terminated when $a / b$ $=0.95$.

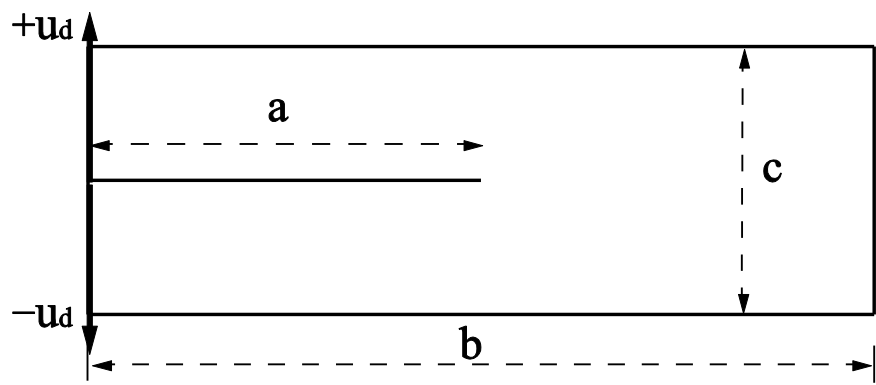

Figure 13. The geometry of double cantilever beam. 
Figure 14 presents the change of energy of $i$ XFEM as crack propagation with both consistent and lumped mass matrices. The unbalanced energy is calculated by $W_{\text {unblance }}$ $=K+W+F-W_{\text {ext }}$, where $K$ is the kinetic energy, $W$ is the strain energy, $F$ is the fracture energy, i.e., the energy consumed for generating new crack surfaces, and $W_{\text {ext }}$ is the total work of external force. It can be seen that the $H$ modification improves the energy conservation for both the consistent and the lumped mass; and the iXFEM without the $H$ modification shows instability for the consistent mass.

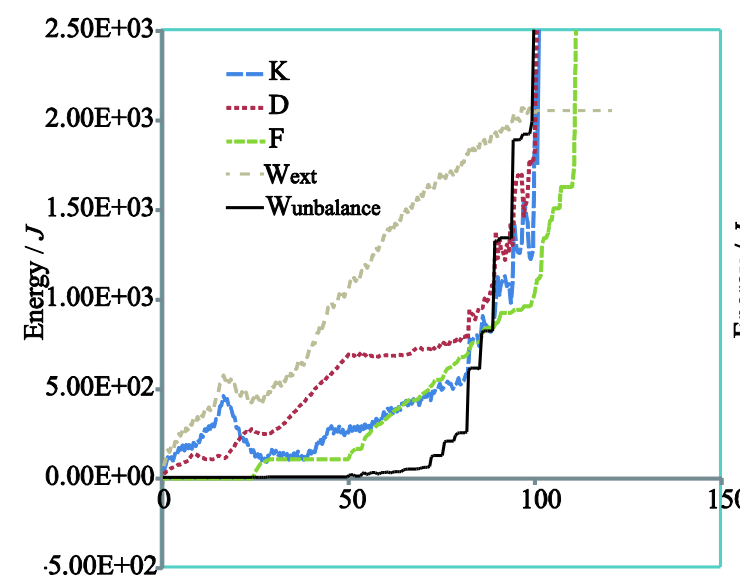

(a)
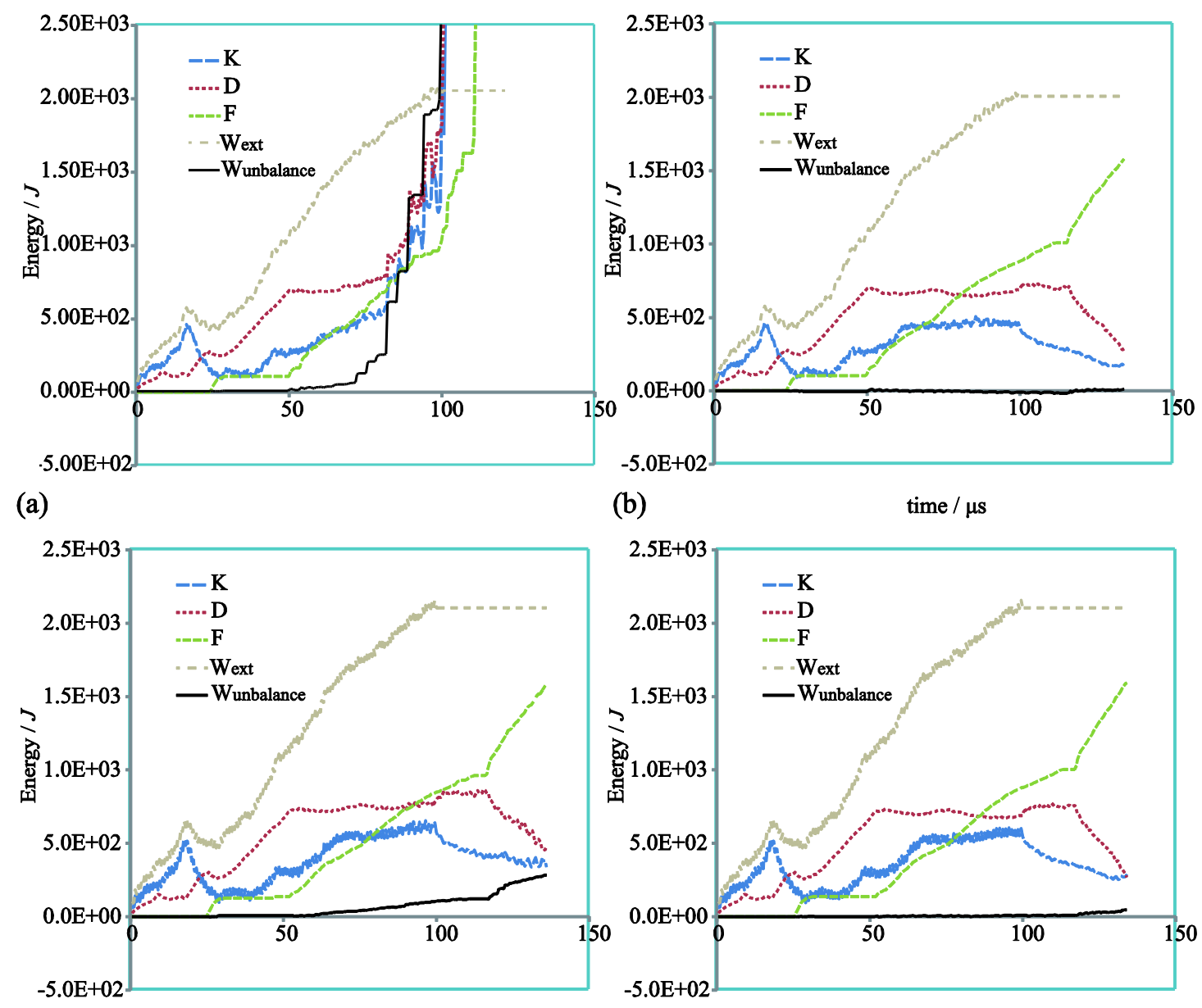

(b)

time / $\mu \mathrm{s}$

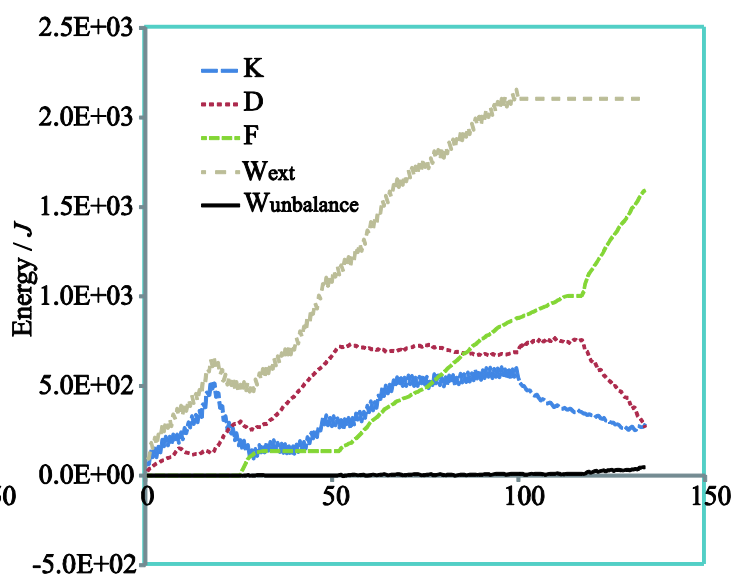

(c)

time / $\mu \mathrm{s}$

(d)

time $/ \mu \mathrm{s}$

Figure 14. Energy versus time. Consistent mass (a) without and (b) with the $H$ modification; lumped mass (c) without and (d) with $H$ modification.

Figure 15 presents the change of the unbalanced energy of $i$ XFEM as crack propagation with reference to the data reported in Reference [28][30][53] for FEM, the FEM with energy balance recovery, and XFEM. It is shown that the unbalanced energy in $i$ XFEM is quite small and it is even smaller than that of the standard FEM and the FEM with the energy balance recovery [53] (respectively labelled as "FEM" and "FEM with balance recovery" in the figure). The "XFEM" of Figure 15 shows the best energy conservation but the good accuracy in the energy conservation does not result in the same good accuracy in the SIF calculation, as will be seen in the next example. 


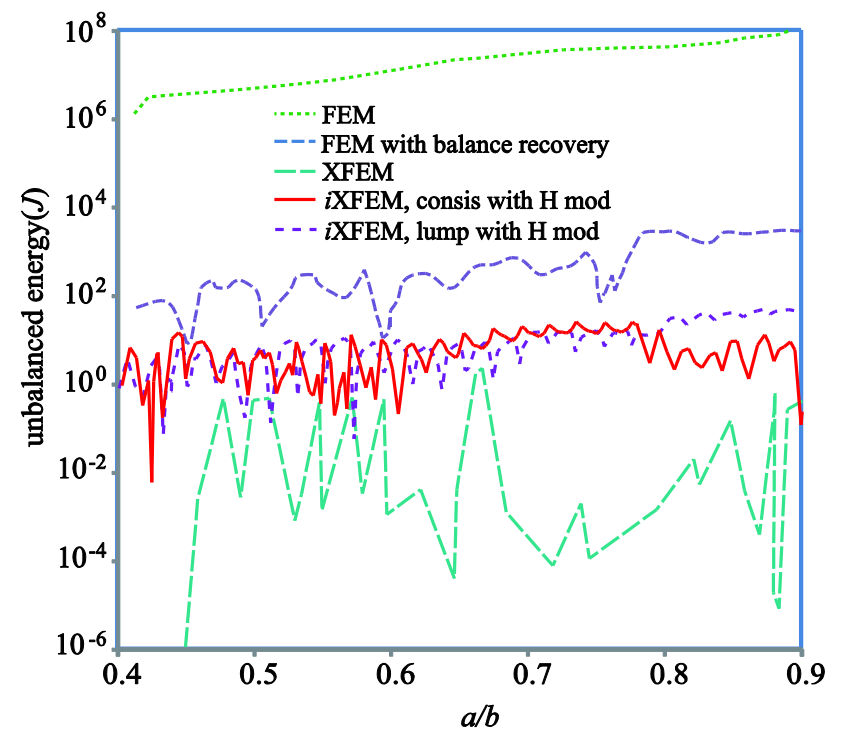

Figure 15. Unbalanced numerical energy as crack propagation.

7.4 Accuracy of dynamic stress intensity factor: stationary and then moving mode I semi-infinite crack

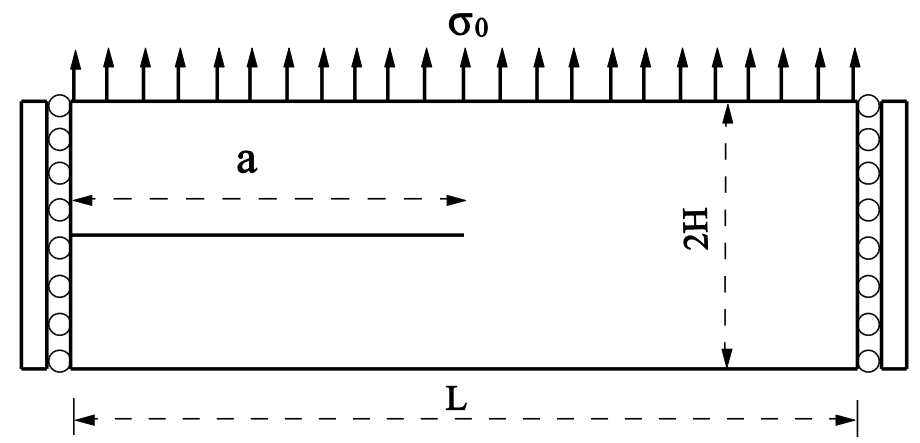

Figure 16. Geometry and loading for mode I semi-infinite crack problem.

The infinite plate with an edge crack is a popular benchmark problem for dynamic crack growth analysis and has been extensively referred [22]-[28][32]-[34]. The problem setup is illustrated in Figure 16. $L=10 \mathrm{~m}, H=2 \mathrm{~m}, a=5 \mathrm{~m}, \rho=8000 \mathrm{~kg} / \mathrm{m}^{3}, E=210$ $\mathrm{GPa}, v=0.3, \sigma_{0}=500 \mathrm{kPa}$. Plain strain is assumed. The analytical solution to the dynamic stress intensity factor of the problem is given by Freund (1990) [45],

$$
K_{\mathrm{I}}^{\mathrm{dyn}}(\dot{a}, t)=k(\dot{a}) K_{\mathrm{I}}^{\mathrm{dyn}}(0, t)
$$

where

$$
k(\dot{a})=\frac{1-\dot{a} / c_{r}}{1-\dot{a} / 2 c_{r}}
$$




$$
K_{\mathrm{I}}^{\mathrm{dyn}}(0, t)= \begin{cases}0 & t<t_{c} \\ \frac{2 \sigma_{0}}{1-\mu} \sqrt{\frac{c_{d}\left(\mathrm{t}-\mathrm{t}_{c}\right)(1-2 \mu)}{\pi}} & t \geq t_{c}\end{cases}
$$

$\dot{a}$ is the propagating velocity of the crack tip, $t_{\mathrm{c}}=H / c_{\mathrm{d}}$ is the elapsed time required for the stress wave to reach the crack tip, $c_{\mathrm{d}}$ is the dilatational wave speed, $\mu$ is the second Lamé constant. Since the geometric is finite, we can only compare the results with the analytical solution until the tensile stress wave is reflected on the bottom side and reaches again the crack tip. Therefore, the simulation time is limited to $t \leq 3 t_{c}=1.009 \times 10^{-3} \mathrm{~s}$.

\section{Case I: Stationary crack}

A uniform mesh of $39 \times 79$ is used for tests. The time step size is chosen as $\Delta t=0.5 \Delta t_{\mathrm{fem}}^{\text {lump }}=7.5 \mu \mathrm{s}$, and the physical time is $t=1 \times 10^{-3} \mathrm{~s}$. The size of $\Omega^{\text {tip }}$ is taken to be $1.0 h$ and the size of the $J$-domain to be $2.0 h$, where $h$ is the mesh size. The size of enrichment domain, the size of the $J$-domain and quadrature are kept the same for both XFEM and $i$ XFEM. The results are plotted in Figure 17, where the stress intensity factors are normalized by $\sigma_{0} \sqrt{H}$ and the time is normalized by $t_{c}$. iXFEM shows the similar accuracy to XFEM for consistent mass but it shows obvious improvement over XFEM for lumped mass. In the test, the only difference between the two methods is the tip enrichment strategy. This example reveals that the mass lumping in $i$ XFEM, while standard and straightforward, leads to better accuracy. In contrast, an "optimal" mass lumping for the tip enrichment of XFEM may still remain.

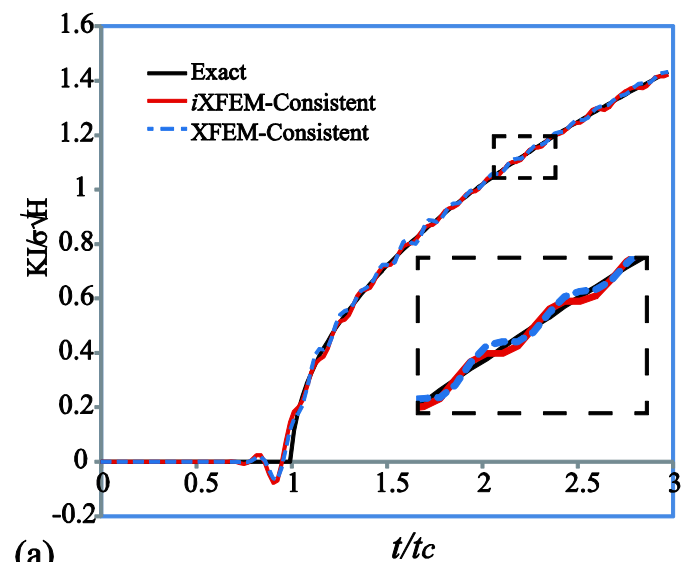

(a)

Figure 17. Accuracy assessment of dynamic SIF for stationary crack.

(a) consistent mass with implicit time intergration, (b) lumped mass with explicit time intergration.

Cases II: Stationary and then moving crack

The crack is kept stationary till $t=1.5 t_{c}$ and then moves in the constant of velocity. The accuracy of dynamic stress intensity factor is evaluated. Four meshes are considered, which are of $19 \times 39,39 \times 79,59 \times 119,99 \times 199$ elements. The lumped mass with explicit time integration is used. The rest parameters are the same as the stationary case. 
The known challenge in this benchmark problem is that the numerical dynamic stress intensity factor presents oscillations and the error introduced by the oscillation is unacceptable [25]. We collected in Figure 18 the current XFEM's solution available in the literature [23][25][26][32][33] and performed a comprehensive comparison. Among those solution of XFEM's, the result reported in Reference [26] presents the least oscillation. A separate comparison with Reference [26] is given in Figure 19.

Among all those solution, iXFEM gives the most accurate solution and the magnitude of oscillation of the $i$ XFEM solution keeps the same during the whole process of crack propagation.

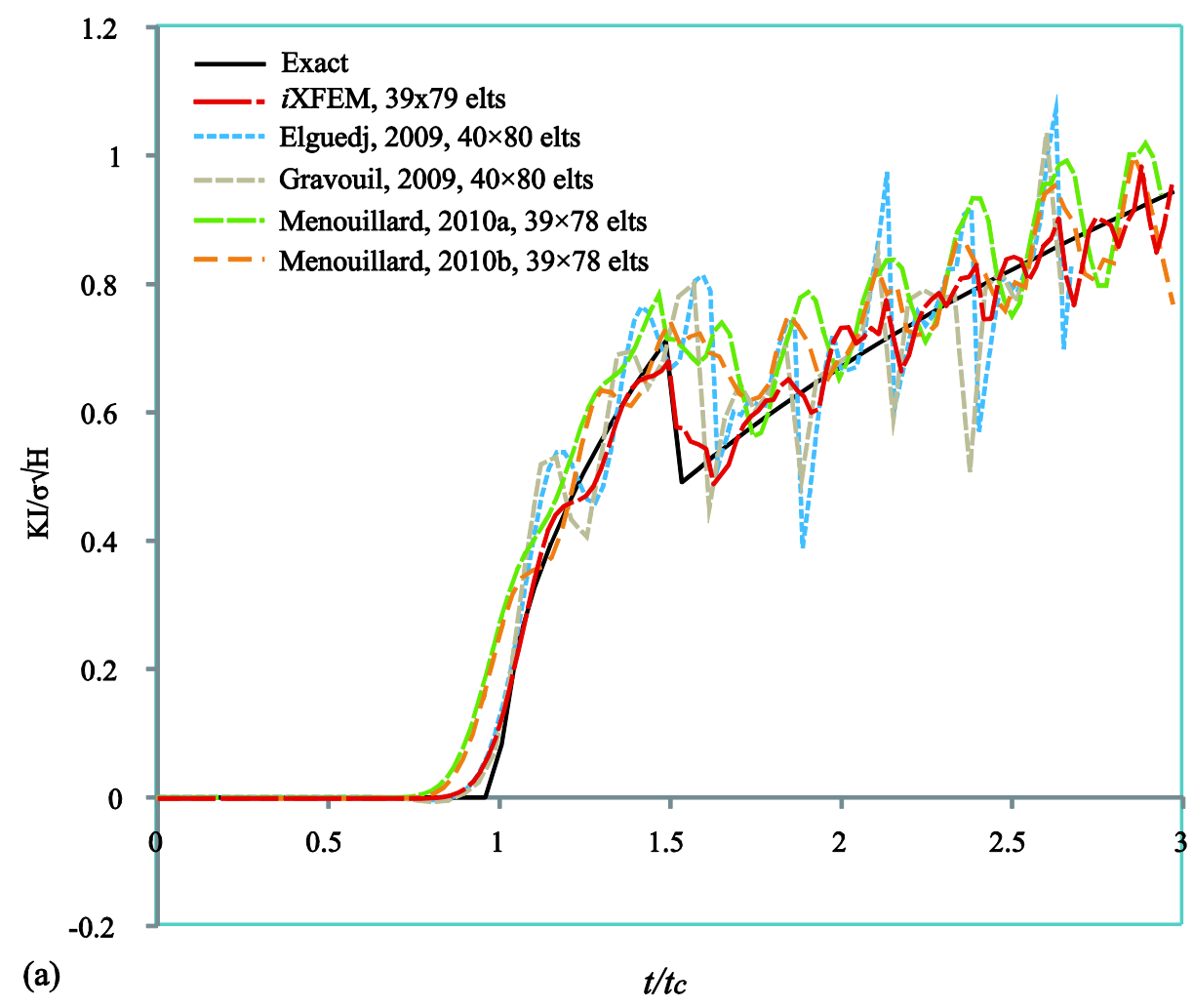




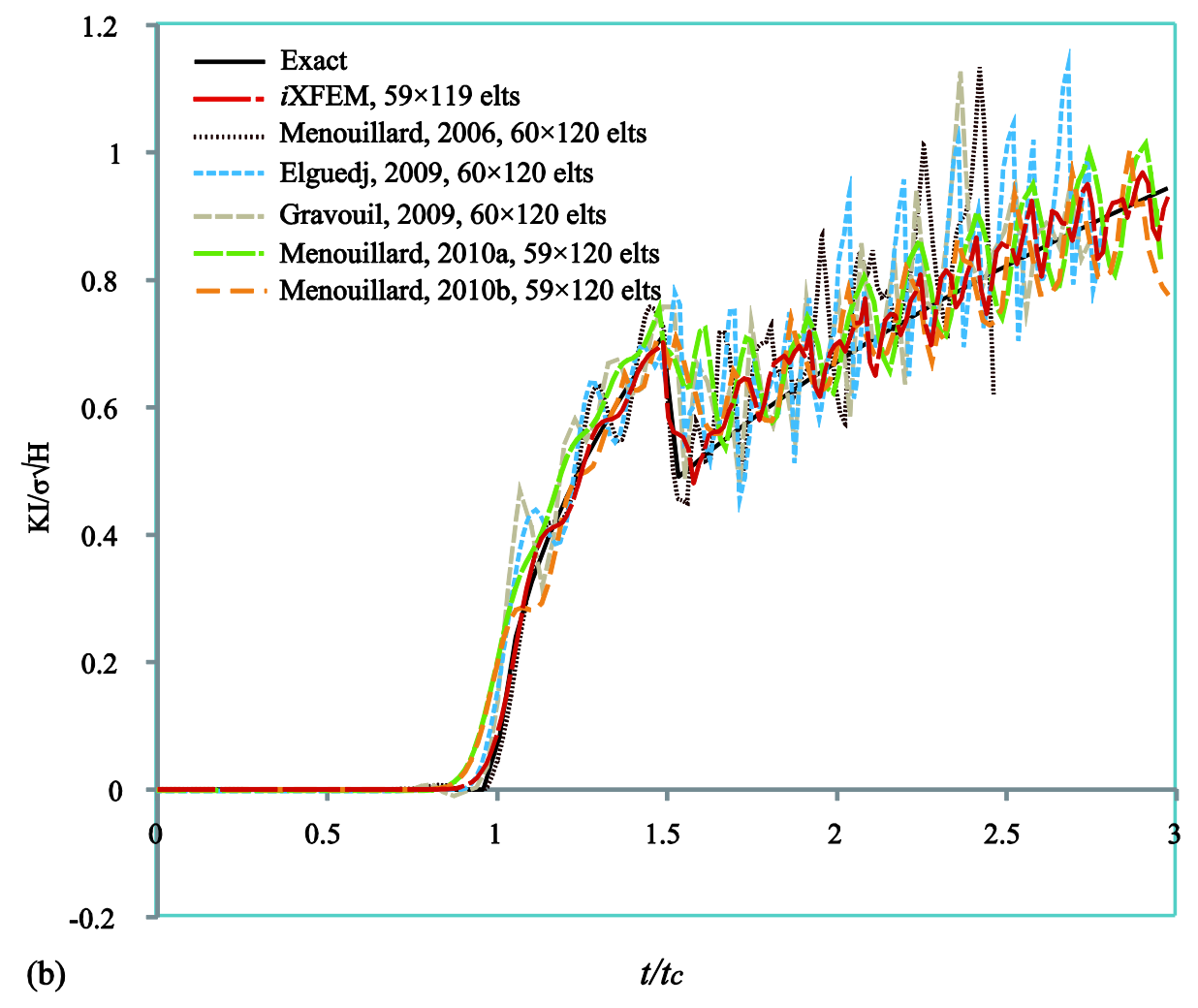

Figure 18. Accuracy assessment of dynamic SIF for moving crack with explicit time integrator (crack starts to move at $t=1.5 t_{\mathrm{c}}$ ). (a) coarse mesh, (b) fine mesh.
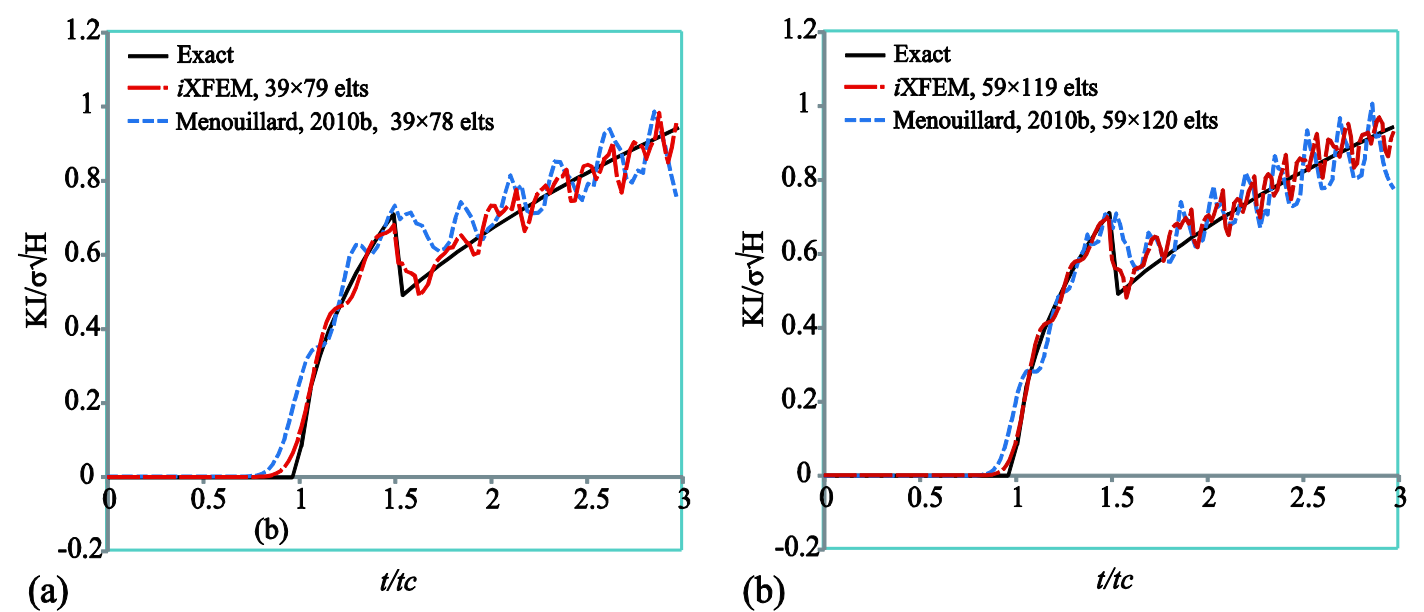

Figure 19. Separate comparisons with Menouillard et al. 2010b [26]). (a) coarse mesh, (b) fine mesh.

In order to clarify the major reason behind the good accuracy of $i$ XFEM, we carried out the following two tests.

First, the COD incompatibility correction, i.e., the $H$ modification, is tested separately on the mesh of $39 \times 79$. The $i$ XFEMs with and without the $H$ modification are compared in Figure 20 (a) and a comparsion with Reference [26], the best solution up to now in literature, is given in Figure 20(b). It is seen that the COD incompatibility correction does reduce the oscillation and further improves accuracy. 
Second, the influence of Gauss quadrature on the accuracy of interaction integral is tested on meshes $39 \times 79$ and $59 \times 119$ using the $2 \times 2$ up to the $8 \times 8$ point quadrature schemes in the J-domain element. As shown in Figure 21, the number of Gauss points shows little effect on the accuracy of SIFs. This verifies that the good accuracy of SIFs is mainly due to the new approximation of $i$ XFEM rather than the scheme of numerical quadrature.

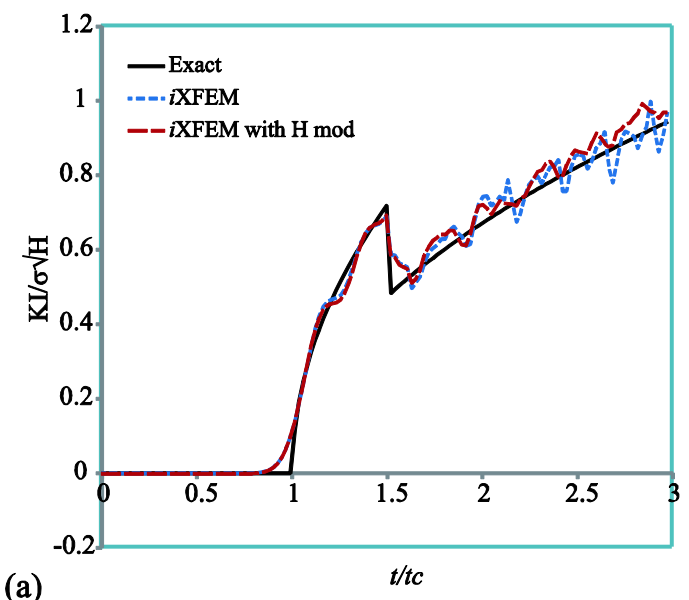

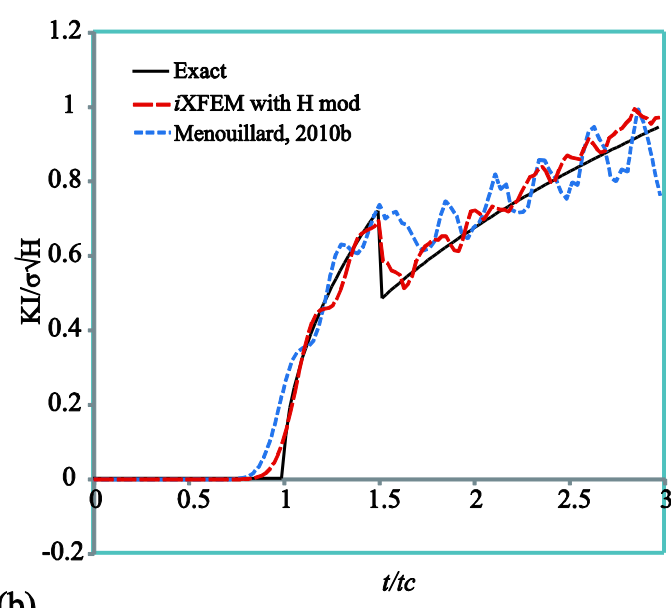

(b)

Figure 20. Effects of COD incompatibility correction. (a) $i$ XFEMs with and without $H$ modifications, (b) Compared with Menouillard et al. 2010b [26].

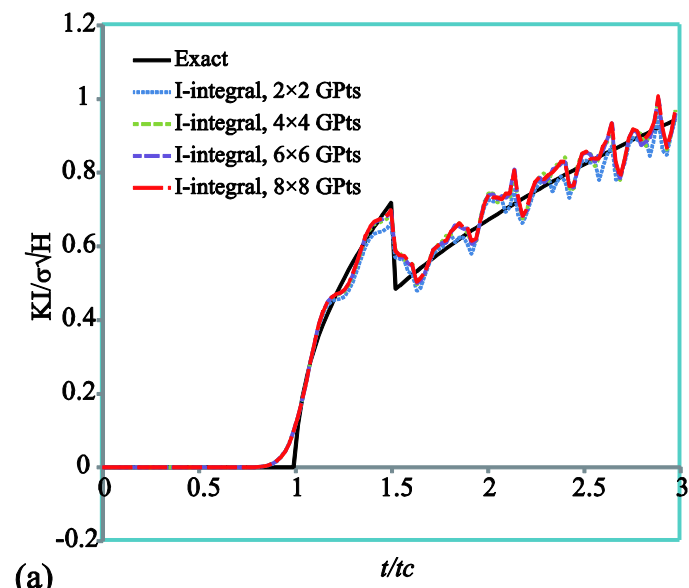

(a)

Figure 21. Accuracy assessment of dynamic SIF for moving crack with different Gauss points in $J$-domain element of interaction integral. (a) coarse mesh, (b) fine mesh. 


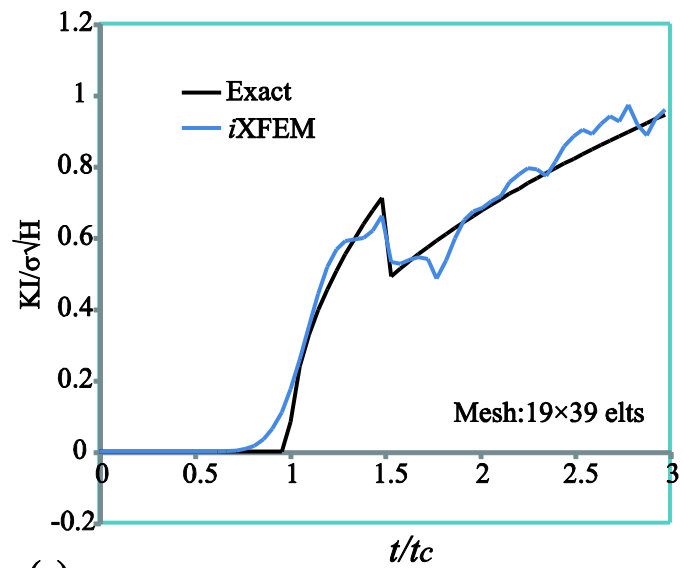

(a)

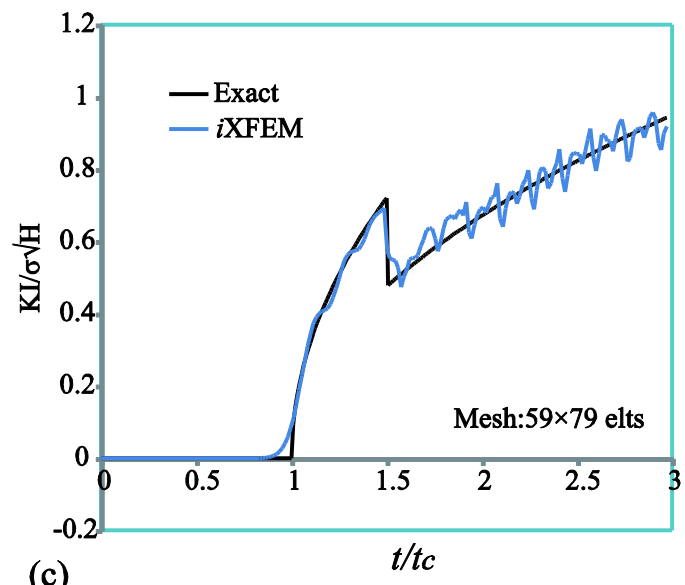

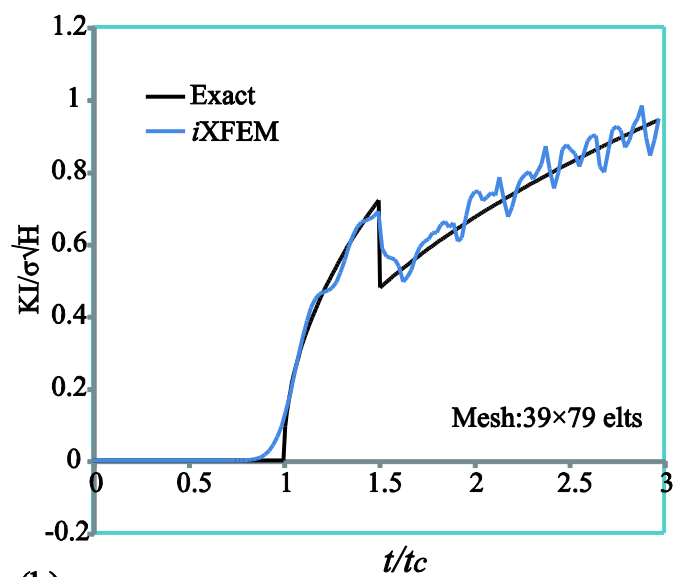

(b)

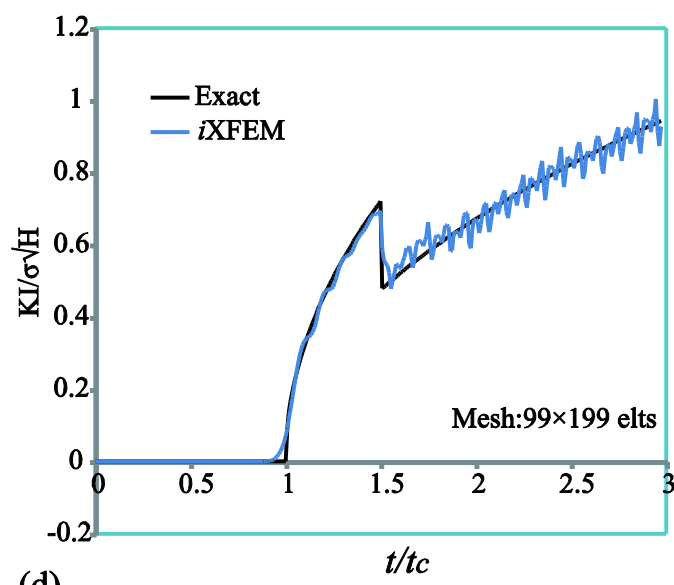

(d)

Figure 22. Accuracy of dynamic SIF versus mesh refinement.

Finally, the effect of mesh refinement is examined in Figure 22. Two facts are observed: (1) the mesh refinement does not smooth out the oscillation; (2) for $i$ XFEM, the amplitude of oscillation almost keeps at a constant level as the crack propagation.

In order to further investigate the source of the oscillation, we fix $\Omega^{\text {tip }}$ to be $1 \mathrm{~m} \times 1 \mathrm{~m}$ and does not relocate along with crack growth. As such, no Heaviside enrichment is added during the crack growth simulation and the mesh contains only the standard translational dofs and the system of dofs is the same during the whole of numerical computation. By this way, we exclude the effect of dof mapping between time steps. The results are labeled "iXFEM-geometrical" in Figure 23 and compared with those in Figure 22 (labeled as "iXFEM-topological"). It is shown that the avoidance of dof mapping does suppress the oscillation to some extents and with reference to the "ideal" case of "no dof mapping", $i$ XFEM with dof mapping also is comparable in accuracy, reflected by the almost same magnitude of oscillation. This implies that the Heaviside enrichment on the newly generated cut element during the crack propagation is the source of the sharp oscillation, which holds true in XFEM, but the new crack tip enrichment presented in $i$ XFEM reduces the oscillation. 

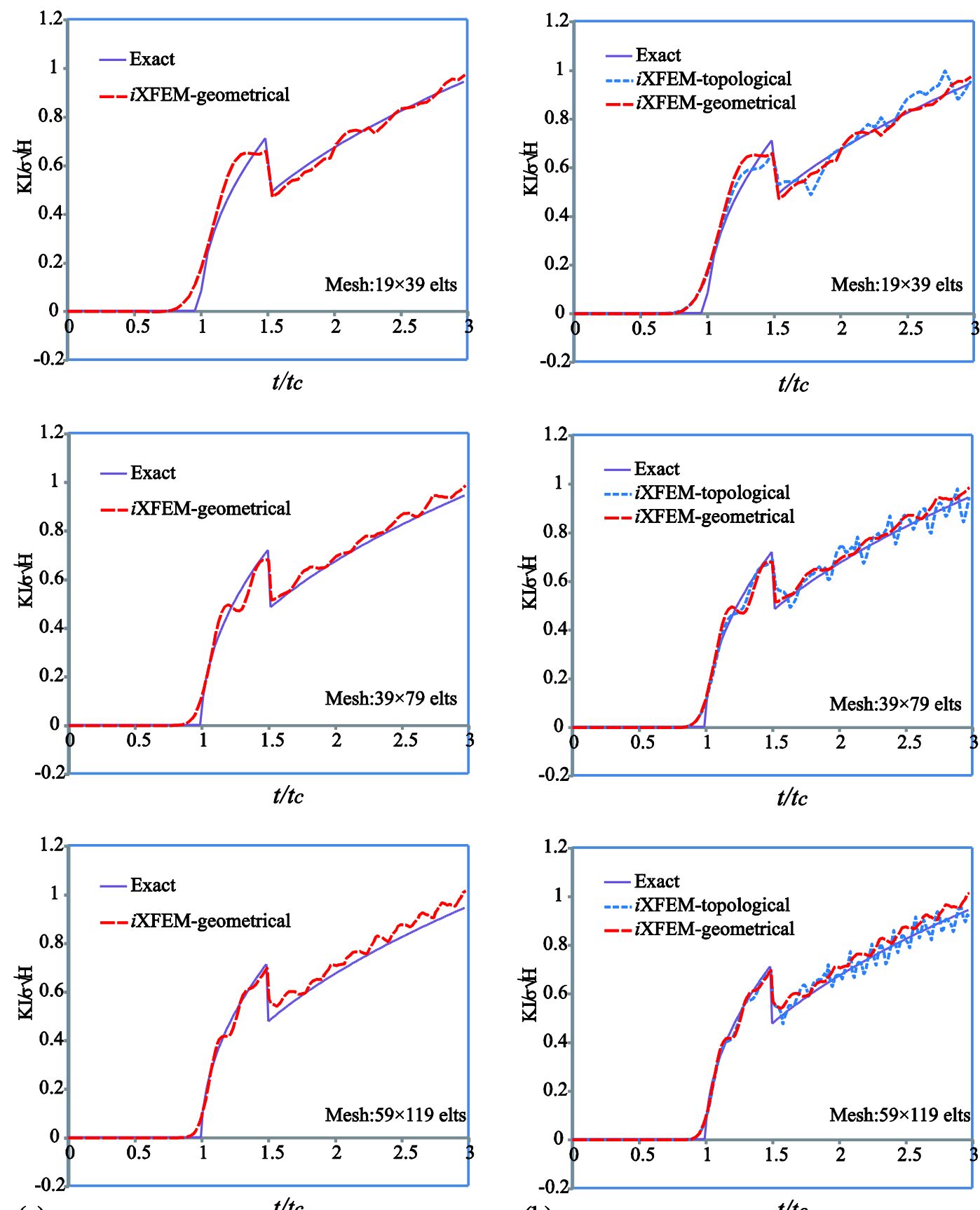

(a)

$$
t / t c
$$

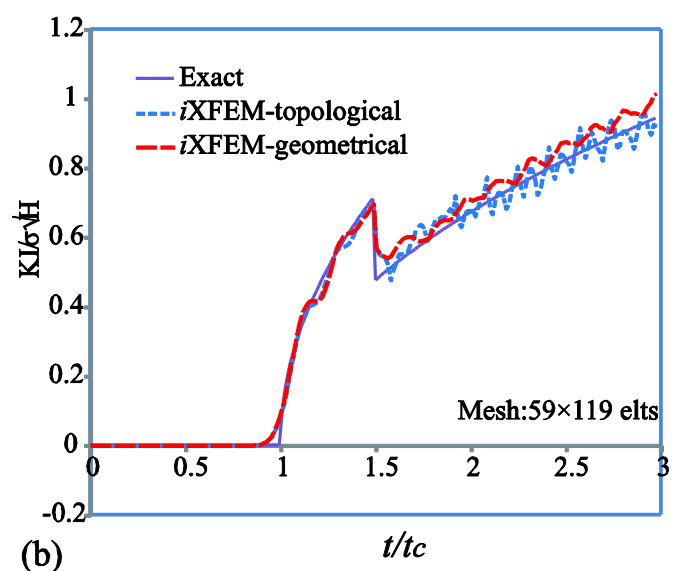

Figure 23. Fixed tip enrichment domain ("iXFEM-geometrical") versus moving tip enrichment domain ("iXFEM-topological").

\subsection{Robustness comparisons with XFEM}

The energy conservative nodal enrichment in the standard XFEM for dynamic problems introduces an increasing number of extra dofs as crack propagating [28]. This leads to elevated bad conditioning in an implicit transient analysis. We compare the two methods in Table 2 for the problem in Figure 16 with a mesh of $39 \times 79$ elements with critical time step $\Delta t=50 \mu \mathrm{s}$. The test is performed for the implicit Newmark method. The conjugate gradient method is used to solve the linear system. Convergence 
tolerance is set to be $10^{-16}$. A computer with eight core of $3.3 \mathrm{GHz}$ and $4 \mathrm{~GB}$ memory are used for the test. The benchmark is designed to reflect the application scenario of $i$ XFEM to large-scale problems in which an iterative solver is the de-facto solver type. Due to the bad conditioning of XFEM, following the common practice, preconditioning is used to solve the linear system of XFEM. Precisely, the SSOR pre-conditioner embedded in Matlab is used. As it is well conditioned, $i$ XFEM is solved by the standard CG solver without pre-conditioning.

It is shown that (1) once the crack starts to move, the conditioning of XFEM fast worsens due to the continuous increase in nodal extra dofs, whereas $i$ XFEM is free of the bad conditioning; The feature makes $i$ XFEM more suitable for an implicit time integrator; (2) although the pre-conditioning can be effective in dealing with the bad conditioning of XFEM in the stationary stage of crack-in this test XFEM even converges faster than $i$ XFEM for $t<1.5 t_{\mathrm{c}}$ thanks to preconditioning, but difficulties arise when conditioning increasingly worsens as the crack propagates. Due to the preconditioning and increasingly worsened conditioning during the crack propagation, $i$ XFEM is several orders of magnitude faster than XFEM in terms of convergence iterations.

Table 2. Robustness comparisons with XFEM.

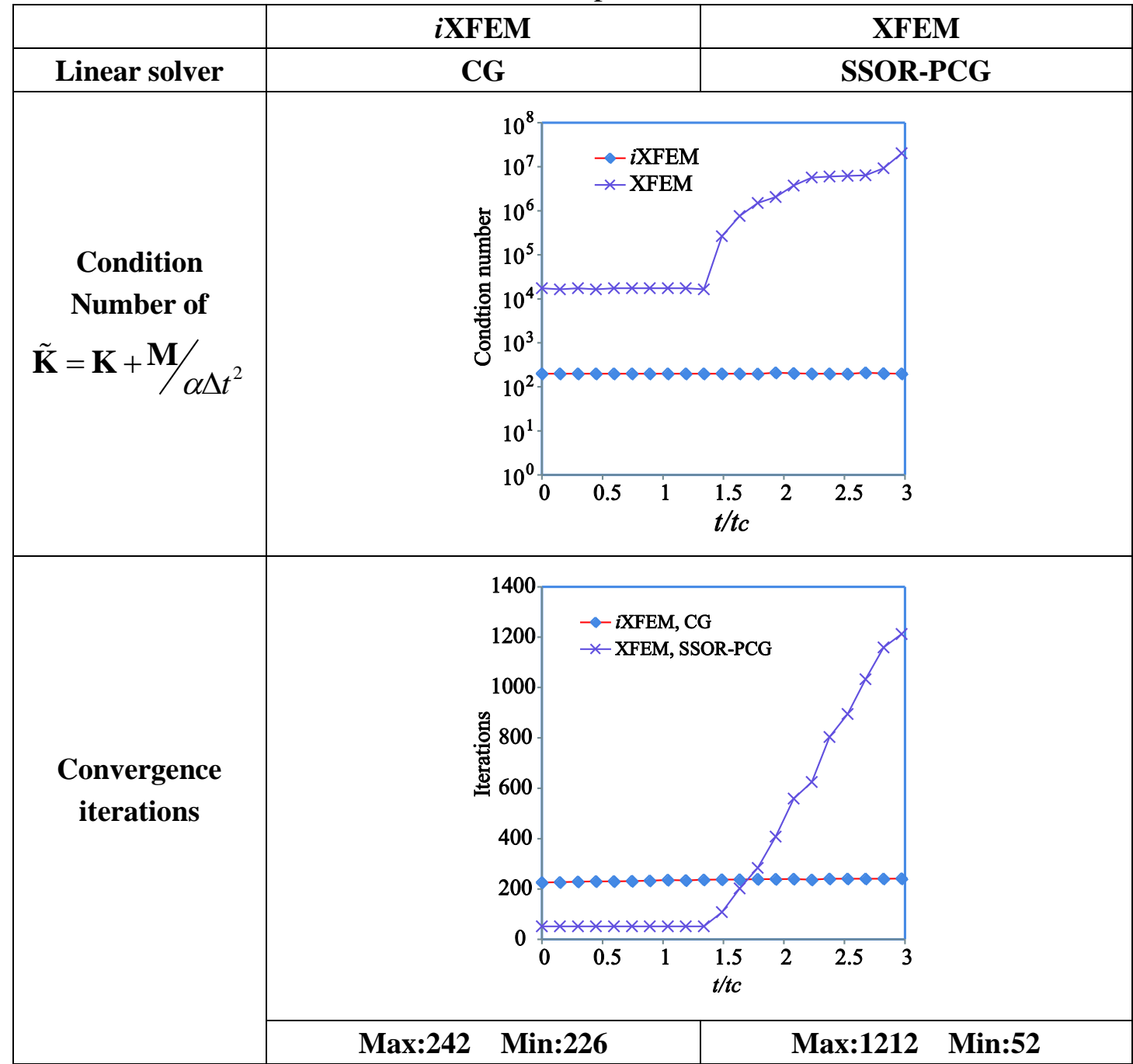




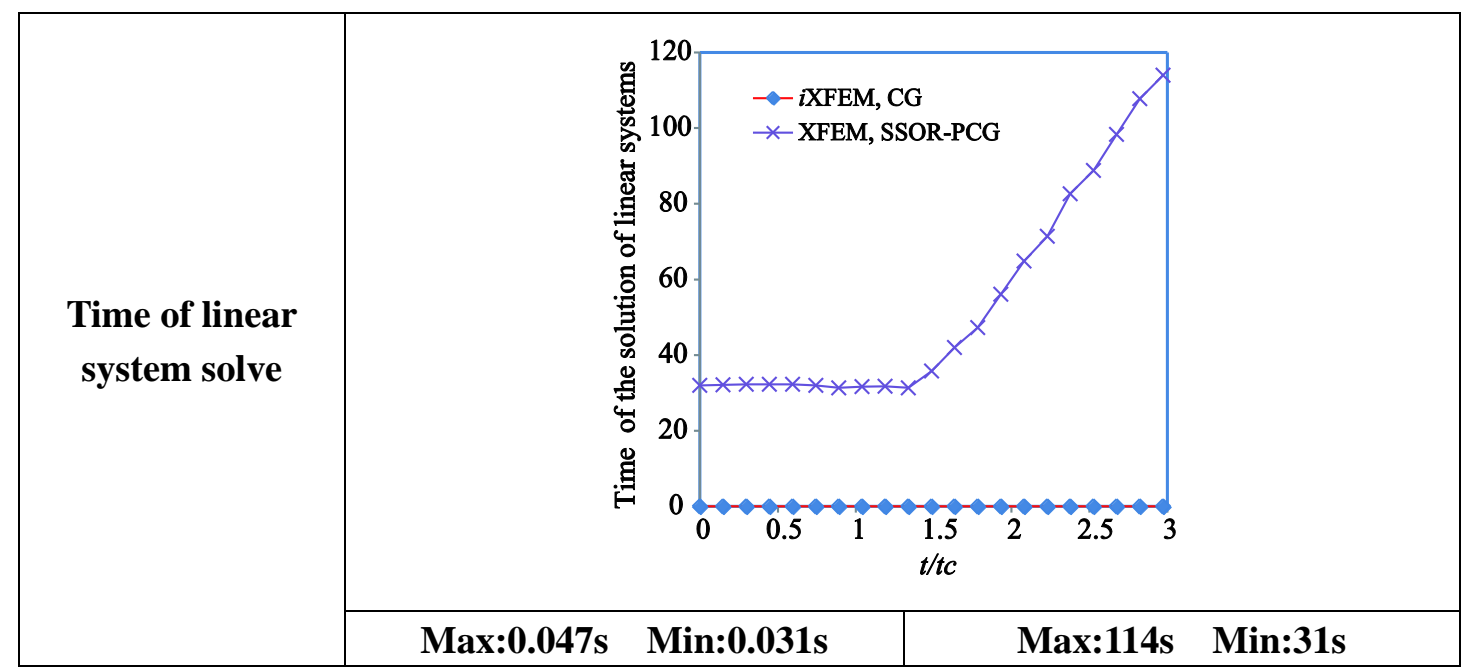

\subsection{Kalthoff's experiment}

The experiment was carried out by Kalthoff [54] in which a maraging steel 18Ni1900 plate with two initial edge notches is impacted by a projectile at speed $\mathrm{V}_{0}$ as shown in Figure 24. By modifying the velocity $\mathrm{V}_{0}$, two different failure modes were observed. At low impact velocity, i.e. under low strain rate, brittle failure with a global angle between $60^{\circ}$ and $70^{\circ}$ is observed; at high velocity, i.e. under high strain rate, a shear band with an angle of $-10^{\circ}$ is observed. Here, we focus on the brittle failure mode with an impact velocity $\mathrm{V}_{0}=20 \mathrm{~m} / \mathrm{s}$. The Kalthoff's experiment has been extensively referred in literature [22][23][25]-[28][32]-[34] for benchmarking the accuracy of crack path prediction using XFEM.

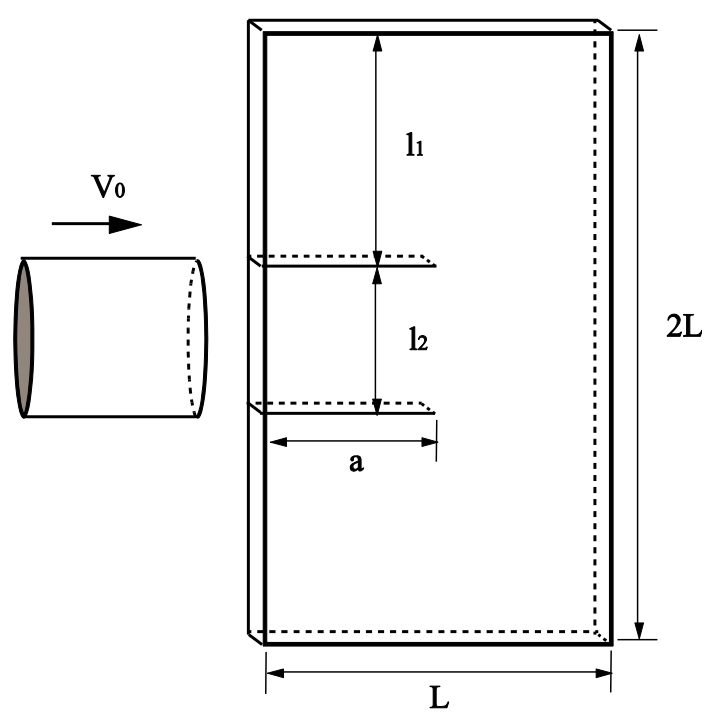

Figure 24. Geometry of Kalthoff's experiment

The geometry of the problem is presented in Figure 24, where $L=100 \mathrm{~mm}, l_{1}=$ $75 \mathrm{~mm}, l_{2}=50 \mathrm{~mm}$, the length of the initial crack $a=50 \mathrm{~mm}$. The material properties of the maraging steel $18 \mathrm{Ni1} 1900$ are: $E=190 \mathrm{GPa}, v=0.3, \rho=8000 \mathrm{~kg} / \mathrm{m}^{3}$ and fracture toughness $K_{\mathrm{IC}}=68 \mathrm{MPa} \sqrt{\mathrm{m}}$. Plain strain is assumed and only the upper half of the plate in experiment are considered. The velocity of crack tip is calculated as follow 
$[25][26][34]:$

$$
\dot{a}= \begin{cases}0 & \text { if } K_{\theta \theta}<K_{1 c} \\ c_{r}\left(1-\left(\frac{K_{1 c}}{K_{\theta \theta}}\right)^{2}\right) & \text { otherwise }\end{cases}
$$

Four uniform meshes $(19 \times 19,39 \times 39,79 \times 79,199 \times 199$ elements $)$ are used for the simulation, and both implicit and explicit time intergrators are tested. The size of $\Omega^{\text {tip }}$ is taken to be $1.0 h$ and the size of the $J$-domain to be $2.0 h$, where $h$ is the mesh size.
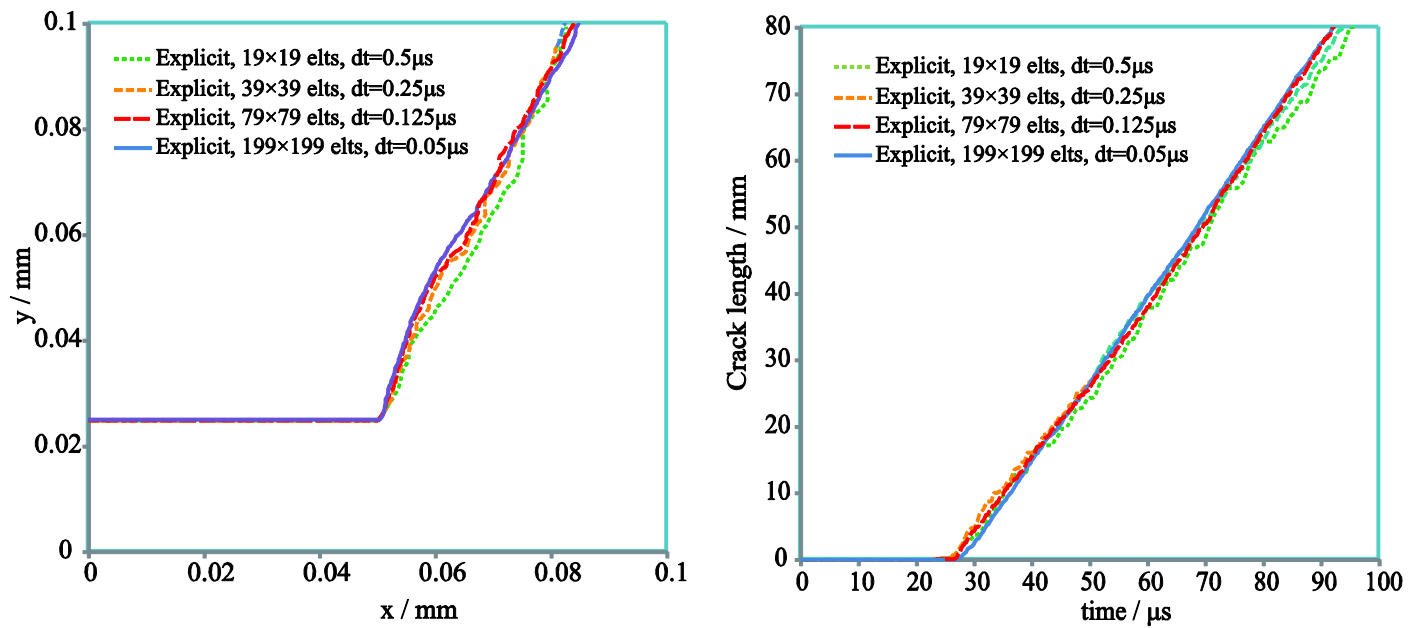

Figure 25. Convergence of predicted crack path and crack length using implicit time integrator. (a) final crack path, (b) crack length versus time.
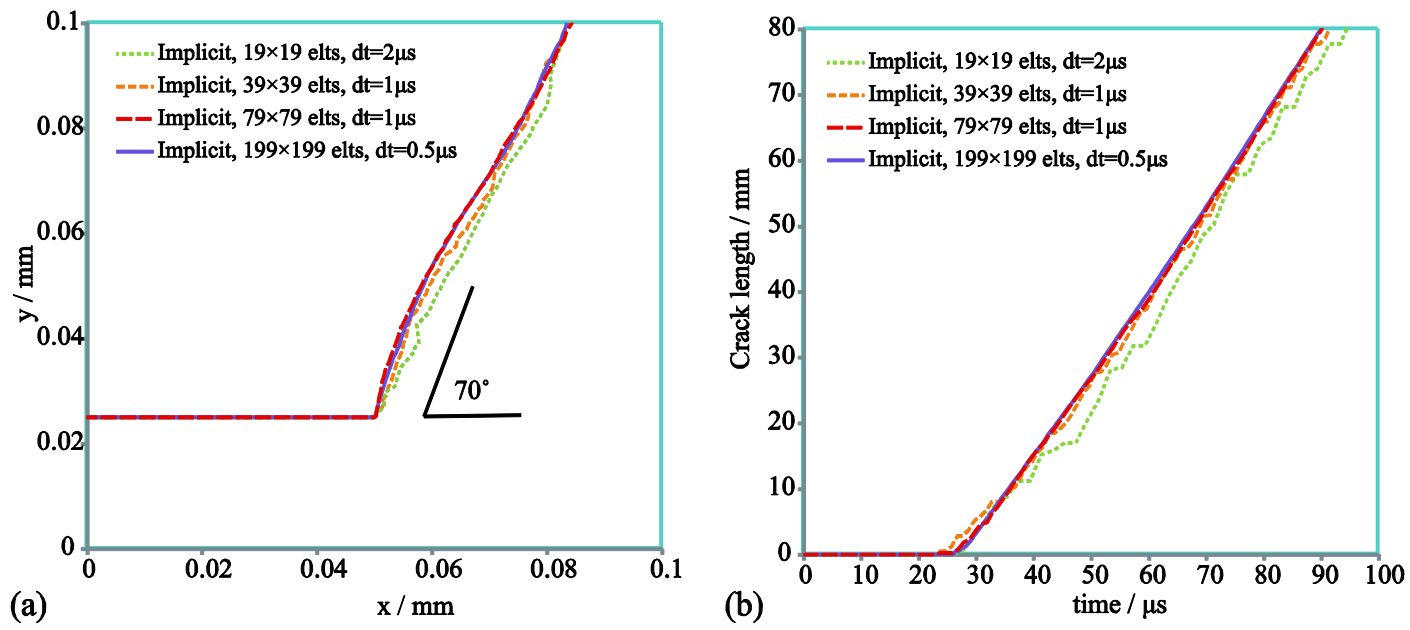

Figure 26. Convergence of predicted crack path and crack length using explicit time integrator. (a) final crack path, (b) crack length versus time. 

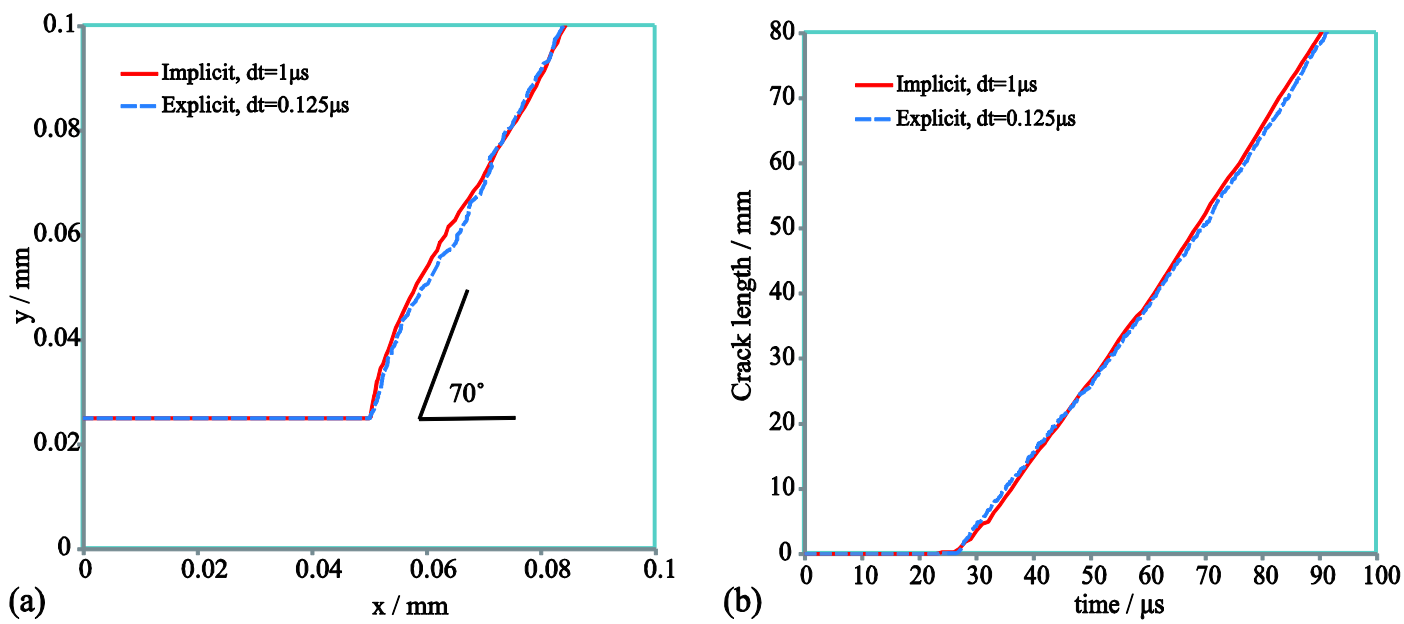

Figure 27. Comparison of the results obtained by implicit and explicit time integrators for the same mesh of $79 \times 79$ elements. (a) final crack path, (b) crack length versus time.

Figures 25 and 26 show the predicted crack path and the crack length, respectively, for implicit and explcit time intergration. A clean comparison between the implicit and the explicit time integration is performed for the mesh $79 \times 79$ in Figure 27.

It is seen that the fine meshes of $79 \times 79$ and $199 \times 199$ give almost the same crack path and the same crack tip velocity, reflecting the convergence of the numerical prediction as mesh refined. The implicit and the explicit integroators also give quite similar prediction. Overall, it is shown that the crack starts to propagate along the direction of around $70^{\circ}$ and ends at about $68^{\circ}$ till the specimen split.

Figure 28 presents the $\sigma_{x}$ stress field and the von Mises stress field at 27, 50, 65, and $80 \mu$ s using the mesh of $199 \times 199$ elements. Several key events during the crack propagation may be understood as follows:

(1) that the crack starts to propagate along the direction of $70^{\circ}$ declined to the horizon at about $27 \mu \mathrm{s}$. This is triggered by the tensile stress (shearing along the pre-crack direction) formed by the elastic wave that has travelled from the left to the right edges, reflected on the right surface, finally reached the crack tip (Figure a);

(2) that the crack path experiences a small deviation at about $50 \mu \mathrm{s}$. This is caused by the compressive stress formed by the elastic wave that has travelled to the left edge, reflected on the left surface and then reached again the crack tip. (Figure b);

(3) that the second tensile stress zone forms around crack tip at about $65 \mu$ s (Figure c);

(4) that thereinafter the crack propagates along the direction of $68^{\circ}$ until the specimen split.

These observation are also in agreement with that reported in References [22][23][25][28][32]-[34].

In the last, we mention that thanks to the well-conditioned global (stiffness) matrix, $i$ XFEM was highly efficient in iterative solve during the above numerical tests. With the good accuracy, the implementation consistency in dynamic analyses, and the solver friendliness, we believe that the improved XFEM is promising in large-scale, multiple crack analyses. 

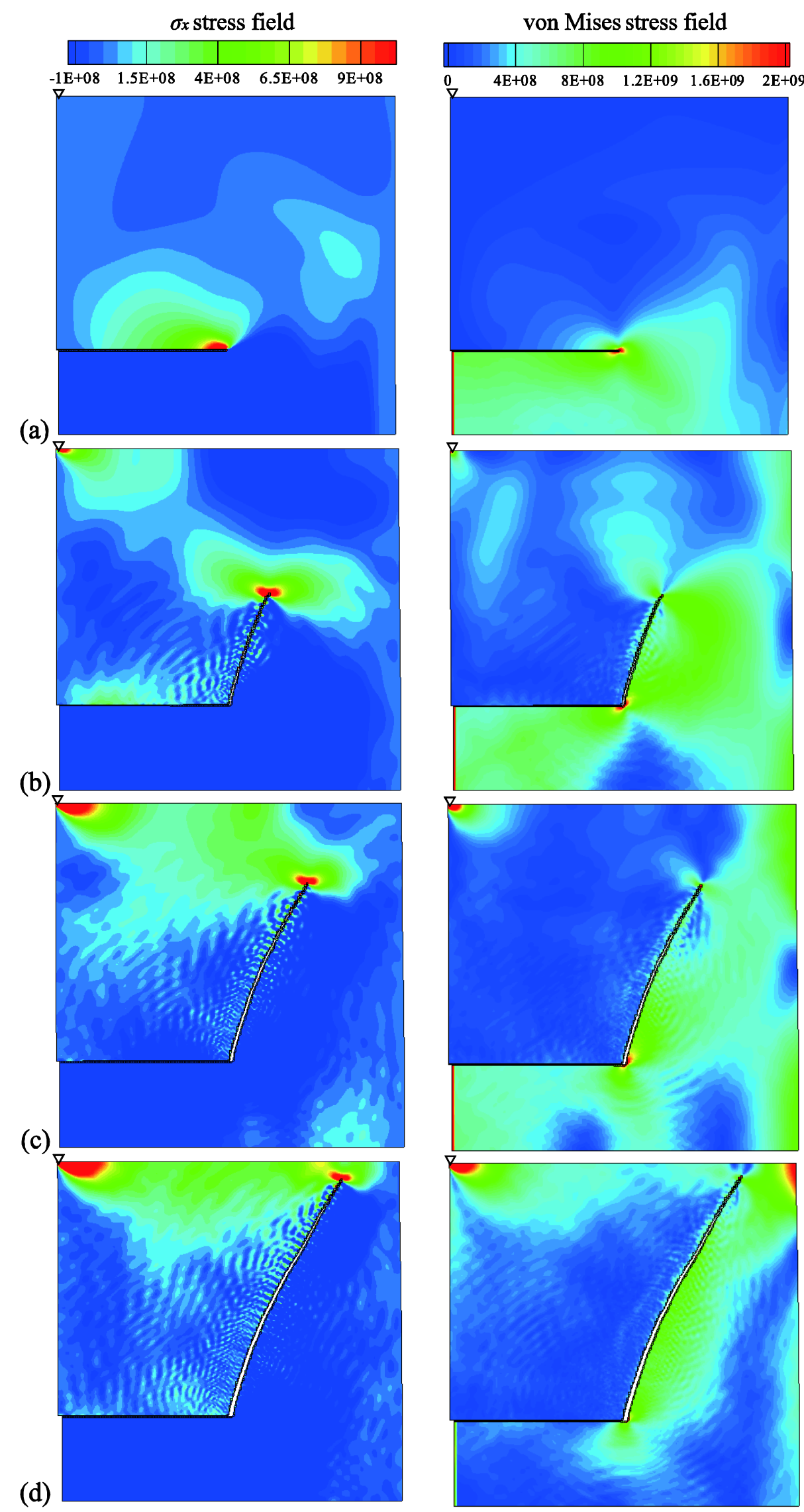

Figure 28. The $\sigma_{x}$ stress field and the Von Mises stress fields obtained for mesh $199 \times 199$ at (a) $25 \mu \mathrm{s}$, (b) $50 \mu \mathrm{s}$, (c) $65 \mu \mathrm{s}$, and (d) $80 \mu \mathrm{s}$. 


\section{Conclusions}

In the development history of XFEM, a direct extension of the singular crack tip enrichment to dynamic/time dependent problems has been a challenge. The newly developed $i$ XFEM overcomes this difficulty. Mass lumping is now standard on the tip enriched element, the critical time step size does not tend to be zero even using the consistent mass, there is no extra-dof dynamics with tip enrichment and hence better energy conservation, conditioning is now the same good as the standard FEM, and remarkably high efficiency is observed with iterative solvers.

Conclusions follow:

(a) For the stationary crack problems tested, $i$ XFEM showed similar accuracy to XFEM for consistent mass but better accuracy than XFEM for lumped mass due to the easy mass lumping;

(b) For the moving crack problems tested, iXFEM showed the best accuracy. In specific, the new method gives the most accurate dynamic stress intensity factors, up to the time the paper published, for the popular yet challenging "mode I semi-infinite stationary and then moving crack" benchmark problem;

(c) For the Conjugate Gradient iterative solver in the implicit time integration, iXFEM was several orders of magnitude faster than XFEM due to the well conditioning property;

(d) For the explicit time integration, the critical time step size of $i$ XFEM for the square element is found to be $2 / 3 \Delta t_{\text {fem }}^{\text {lump }}$, larger than $1 / 2 \Delta t_{\text {fem }}^{\text {lump }}$ of XFEM.

The payoff of $i$ XFEM is the expense on the computation of shape function at the crack tip enriched nodes because the least-squares operation involves matrix inversion. When the crack tip sub-domain is local, this expense would not be dominant. The expense is also compensated by the fast iteration convergence in implicit time integration or the larger critical time step size in explicit time integration. The other issue of $i$ XFEM may arise in parallel implementation, in which the wider bandwidth requires two and more layers of ghost elements and hence more ghost data communication when a crack crosses processor boundaries.

The on-going work includes parallel implementation, engineering application tests and multiple crack interaction analysis at large scale as well as implementations in 3D.

\section{Appendix: Interaction integral and dynamic stress intensity factors}

\section{A.1 Dynamic stress intensity factors}

The stress intensity factor is one of the important parameters representing fracture properties of a crack-tip. In the present study, the dynamic stress intensity factors $K_{\mathrm{I}}$ and $K_{\text {II }}$ are defined by the asymptotic behavior of the stress near crack tip (see Freund [45] for details and reference therein),

$$
\begin{aligned}
K_{\mathrm{I}}^{\mathrm{dyn}} & =\lim _{r \rightarrow 0} \sqrt{2 \pi r} \sigma_{y y}(\theta=0) \\
K_{\mathrm{II}}^{\mathrm{dyn}} & =\lim _{r \rightarrow 0} \sqrt{2 \pi r} \sigma_{x y}(\theta=0)
\end{aligned}
$$


The dynamic stress intensity factors are local quantities defined as limit values, so they cannot be estimated directly and accurately. In 2005, Réthoré et al. [28][51] presented a domain independent integral to calculate dynamic stress intensity factors. The dynamic interaction integral $I^{\text {int }}$ can be obtained from the Lagrangian conservation law using a virtual crack extension field $\mathbf{q}$ as

$$
\begin{aligned}
I^{\text {int }} & =-\int_{A}\left(\left(\boldsymbol{\sigma}^{\text {aux }}: \nabla \mathbf{u}-\rho \dot{\mathbf{u}} \dot{\mathbf{u}}^{\text {aux }}\right) \operatorname{div}(\mathbf{q})-\left(\boldsymbol{\sigma}^{\text {aux }}:(\nabla \mathbf{u} \nabla \mathbf{q})+\boldsymbol{\sigma}:\left(\nabla \mathbf{u}^{\text {aux }} \nabla \mathbf{q}\right)\right)\right) \mathrm{d} S \\
& +\int_{A}\left(\operatorname{div}\left(\boldsymbol{\sigma}^{\text {aux }}\right) \nabla \mathbf{u}(\mathbf{q})+\rho \ddot{\mathbf{u}} \nabla \mathbf{u}^{\text {aux }}(\mathbf{q})+\rho \dot{\mathbf{u}}^{\text {aux }} \nabla \dot{\mathbf{u}}(\mathbf{q})+\rho \dot{\mathbf{u}} \nabla \dot{\mathbf{u}}^{\text {aux }}(\mathbf{q})\right) \mathrm{d} S
\end{aligned}
$$

where $\boldsymbol{\sigma}^{\text {aux }}$ and $\mathbf{u}^{\text {aux }}$ are auxiliary stress and displacement fields, $A$ is the area eaged by any contour $\partial A$ enclosing the crack tip, $\mathbf{q}$ is the virtual crack extension field and is particularized as follows:

$$
q=\left\{\begin{array}{l}
0 \text { on } \partial A \\
1 \text { in } A, \text { tangent to the crack's face }
\end{array}\right.
$$

Then, a dynamic energy analysis provides an equivalent to Irwin's relation in plane strain,

$$
I^{\mathrm{int}}=\frac{2\left(1-v^{2}\right)}{E}\left(f_{1}(\dot{a}) K_{\mathrm{I}}^{\mathrm{dyn}} K_{\mathrm{I}}^{\mathrm{aux}}+f_{2}(\dot{a}) K_{\mathrm{II}}^{\mathrm{dyn}} K_{\mathrm{II}}^{\mathrm{aux}}\right)
$$

where $K_{\mathrm{I}}^{\mathrm{dyn}}$ and $K_{\mathrm{II}}^{\mathrm{dyn}}$ are the stress intensity factors of the auxiliary fields, $f_{i}$ are universal functions of the speed of the crack tip $\dot{a}$,

$$
\begin{aligned}
& f_{i}(\dot{a})=\frac{4 \alpha_{i}\left(1-\alpha_{j}^{2}\right)}{(k+1) D}, \\
& \alpha_{d}=\sqrt{1-\left(\frac{\dot{a}}{c_{d}}\right)^{2}}, \quad \alpha_{s}=\sqrt{1-\left(\frac{\dot{a}}{c_{s}}\right)^{2}}, \\
& D=4 \alpha_{d} \alpha_{s}-\left(1+\alpha_{s}^{2}\right)^{2}
\end{aligned}
$$

where $\kappa$ is the Kolosov coefficient, which is $3-4 v$ for the plane strain, and $(3-v) /(1$ $+v)$ for the plane stress, $c_{d}$ and $c_{s}$ are, respectively, the dilatational and the shear velocities, and are given as a function of the Lamé coefficients and the density,

$$
c_{d}=\sqrt{\frac{\lambda+2 \mu}{\rho}}, \quad c_{s}=\sqrt{\frac{\mu}{\rho}}
$$

$D(\dot{a})$ is a function of the speed of the crack tip $\dot{a}, D(\dot{a})=0$ when the Rayleigh wave speed is equal to $c_{r}$, which is the Rayleigh wave speed and the theoretical maximum velocity of a crack in a homogeneous medium.

The different stress intensity factors can be estimated through an appropriate choice of $\mathbf{u}^{\text {aux }} \cdot K_{\mathrm{I}}^{\text {dyn }}$ is evaluated through $K_{\mathrm{I}}^{\text {aux }}=1, K_{\mathrm{II}}^{\text {aux }}=0$ while $K_{\mathrm{II}}^{\text {dyn }}$ through $K_{\mathrm{I}}^{\mathrm{aux}}=0, K_{\mathrm{II}}^{\mathrm{aux}}=1$. In order to evaluate the stress intensity factors accurately, the interaction integral is calculated using a set of additional integration elements rich in Gauss points, called the $J$-domain [23][28]. The elements of the $J$-domain are used only for the calculation of the stress intensity factors and are independent of the mesh of the body. This $J$-domain follows the crack tip and its orientation as the crack growth. In the paper, 16 square elements each with $8 \times 8$ Gauss points are defined as the $J$-domain.

\section{A.2 Fracture criteria}


The equivalent dynamic stress intensity factor $K_{\theta \theta}$ is defined by [23],

$$
K_{\theta \theta}=\cos ^{3}\left(\frac{\theta_{c}}{2}\right) K_{\mathrm{I}}^{\mathrm{dyn}}-\frac{3}{2} \cos \left(\frac{\theta_{c}}{2}\right) \sin \left(\theta_{c}\right) K_{\mathrm{II}}^{\mathrm{dyn}}
$$

where $\theta_{c}$ is the direction of crack propagation, which is determined by the direction of the maximum hoop stress. The formulation of the direction $\theta_{c}$ is a function of the stress intensity factors,

$$
\theta_{c}=2 \arctan \left(\frac{1}{4}\left[\frac{K_{\mathrm{I}}^{\mathrm{dyn}}}{K_{\mathrm{II}}^{\mathrm{dyn}}}-\operatorname{sign}\left(K_{\mathrm{II}}^{\mathrm{dyn}}\right) \sqrt{8+\left(\frac{K_{\mathrm{I}}^{\mathrm{dyn}}}{K_{\mathrm{II}}^{\mathrm{dyn}}}\right)^{2}}\right]\right)
$$

Then, if the maximum hoop stress intensity factor $K_{\theta \theta}$ is greater than the quasistatic fracture toughness $K_{1 C}$, the crack propagation and the instantaneous maximum hoop stress intensity factor stay equal to the dynamic crack growth toughness $K_{1 D}$ (see Reference [23][52]).

$$
K_{1 D}=\frac{K_{1 C}}{1-\left(\dot{a} / c_{r}\right)^{m}}
$$

By considering $m=1$ and $K_{\theta \theta}=K_{1 D}$, the expression for the crack velocity is obtained,

$$
\dot{a}=\left(1-\frac{K_{1 c}}{K_{\theta \theta}}\right) c_{r}
$$

\section{Acknowledgement:}

The financial support from National Science Foundation of China (grand \# 11472274) is acknowledged.

\section{References:}

[1] T. Belytschko, T. Black, Elastic crack growth in finite elements with minimal remeshing, Int. J. Numer. Methods Engrg. 45 (1999) 601-620.

[2] N. Moes, J. Dolbow, T. Belytschko, A finite element method for crack growth without remeshing, Int. J. Numer. Methods Engrg. 46 (1999) 131-150.

[3] T. Belytschko, N. Moes, S. Usui, C. Parimi, Arbitrary discontinuities in finite elements., Int. J. Numer. Methods Engrg. 50 (2001) 993-1013.

[4] A. Gravouil, N. Moes, T. Belytschko, Non-planar 3D crack growth by the extended finite element and level sets-Part II: Level set update, Int. J. Numer. Methods Engrg. 53 (2002) 2569-2586.

[5] A. Afshar, A. Daneshyar, S. Mohammadi, XFEM analysis of fiber bridging in mixed-mode crack propagation in composites, Compos. Struct. 125 (2015) 314-327.

[6] Y. Shen, A.J. Lew, A locking-free and optimally convergent discontinuous- Galerkin-based extended finite element method for cracked nearly incompressible solids, Comput. Methods Appl. Mech. Engrg. 273 (2014) 119-142.

[7] T.Q. Bui, C. Zhang, Analysis of generalized dynamic intensity factors of cracked magnetoelectroelastic solids by XFEM, Finite Elements in Analysis and Design. 69 (2013) $19-36$. 
[8] R. Pourmodheji, M. Mashayekhi, Improvement of the extended finite element method for ductile crack growth, Materials Science and Engineering A. 551 (2012) 255- 271.

[9] I.V. Singh, B.K. Mishra, S. Bhattacharya, R.U. Patil, The numerical simulation of fatigue crack growth using extended finite element method, Int. J. Fatigue. 36 (2012) 109-119.

[10] T. Belytschko, R. Gracie, G. Ventura, A review of extended/generalized finite element methods for material modeling, Modelling Simul. Mater. Sci. Eng. 17 (2009) 043001.

[11] T.P. Fries, T. Belytschko, The extended/generalized finite element method: An overview of the method and its applications, Int. J. Numer. Methods Engrg. 84 (2010) 253-304.

[12] http://www.3ds.com/products-services/simulia/portfolio/abaqus/latest-release/

[13] I. Babuška, J.M. Melenk, Partition of unity method, Int. J. Numer. Methods Engrg. 40 (1997) $727-758$.

[14] J.M. Melenk, I. Babuška, The partition of unity finite element method: basic theory and applications, Comput. Methods Appl. Mech. Engrg. 139 (1996) 289 -314.

[15] R. Tian, G. Yagawa, H. Terasaka, Linear dependence problems of partition of unity based generalized FEMs, Comput. Methods Appl. Mech. Engrg. 195 (2006) 4768-4782.

[16] E. Béchet, H. Minnebo, N. Moës, B. Burgardt, Improved implementation and robustness study of the x-fem for stress analysis around cracks, Int. J. Numer. Methods Engrg. 64 (2005) 1033-1056.

[17] P. Laborde, J. Pommier, Y. Renard, M. Salaun, High-order extended finite element method for cracked domains, Int. J. Numer. Methods Engrg. 64 (2005) 354-381.

[18] E. Chahine, P. Laborde, Y.A. Renard, Crack tip enrichment in the XFEM using a cutoff function, Int. J. Numer. Methods Engrg. 75 (2008) 629-646.

[19] I. Babuska, U. Banerjee, Stable generalized finite element method, Comput. Methods Appl. Mech. Engrg. 201-204 (2012) 91-111.

[20] V. Gupta, C.A. Duarte, I. Babuska, U. Banerjee, A stable and optimally convergent generalized FEM (SGFEM) for linear elastic fracture mechanics, Comp. Methods Appl. Mech. Engrg. 266 (2013) 23-39.

[21] V. Gupta, C.A. Duarte, I. Babuska, U. Banerjee, Stable GFEM (SGFEM): Improved conditioning and accuracy of GFEM/XFEM for three-dimensional fracture mechanics, Comp. Methods Appl. Mech. Engrg. 289 (2015) 355-386.

[22] T. Belytschko, H. Chen, J. Xu, G. Zi, Dynamic crack propagation based on loss of hyperbolicity and a new discontinuous enrichment, Int. J. Numer. Methods Engrg. 58 (2003) 1873-905.

[23] T. Menouillard, J. Réthoré, A. Combescure, H. Bung, Efficient explicit time stepping for the extended finite element method, Int. J. Numer. Methods Engrg. 68 (2006) 911-938.

[24] T. Menouillard, J. Réthoré, N. Moës, A. Combescure, H. Bung, Mass lumping strategies for X-FEM explicit dynamics: Application to crack propagation, Int. J. Numer. Methods Engrg. 74 (2008) 447-474.

[25] T. Menouillard, J. Song, Q. Duan, T. Belytschko, Time dependent crack tip enrichment for dynamic crack propagation, Int. J. Fract. 162 (2010a) 33-49.

[26] T. Menouillard, T. Belytschko, Smoothed nodal forces for improved crack propagation modeling in XFEM. Int J for Num. Methods in Eng. 84(2010b) 47-72.

[27]T. Menouillard, T. Belytschko. Dynamic fracture with meshfree enriched XFEM. Acta Mechanica. 213(2010c) 53-69.

[28] J. Réthoré, A. Gravouil, A. Combescure, An energy-conserving scheme for dynamic crack growth using the extended finite element method, Int. J. Numer. Methods Engrg. 63 (2005) 631-659.

[29] B. Prabel, A. Combescure, A. Gravouil, et al, Level set X-FEM non matching meshes: application to dynamic crack propagation in elastic-plastic media, Int. J. Numer. Methods Engrg. 69 (2007) 1553-1569.

[30] A. Combescure, A. Gravouil, D. Gregoire, J. Réthoré, XFEM a good candidate for energy conservation in simulation of brittle dynamic crack propagation, Comp. Methods Appl. Mech. Engrg. 197 (2008) 309-318.

[31] R. de Borst, J.J.C. Remmers, A. Needleman, Mesh independent discrete numerical representations of cohesive-zone models, Engrg. Fract. Mech. 73 (2006) 160-177. 
[32] T. Elguedj, A. Gravouil, Maigre H, An explicit dynamics extended finite element method. Part 1: mass lumping for arbitrary enrichment functions, Comp. Methods Appl. Mech. Engrg. 198 (2009) 2297-2317.

[33] A. Gravouil, T. Elguedj, H. Maigre, An explicit dynamics extended finite element method. Part 2: Element-by-element stable-explicit/explicit dynamic scheme, Comp. Methods Appl. Mech. Engrg. 198 (2009) 2318-2328.

[34] Z.L. Liu, T. Menouillard, T. Belytschko. An XFEM/Spectral element method for dynamic crack propagation, Int. J. Fract. 169 (2011) 183-198.

[35] A. Combescure, A. Gravouil, D. Gregoire, J. Réthoré, X-FEM a good candidate for energy conservation in simulation of brittle dynamic crack propagation. Comput. Methods Appl. Mech. Engrg. 197(2008) $309-318$.

[36] T. Belytschko, D. Organ, Y. Krongauz. A coupled finite element-element-free Galerkin method, Comp. Mech. 17 (1995) 186-195.

[37] T.P. Fries, T. Belytschko. The intrinsic partition of unity method. Comp. Mech. 40 (2007) 803-814.

[38] R. Tian, Extra-dof-free and linearly independent enrichments in GFEM, Comp. Methods Appl. Mech. Engrg. 266 (2013) 1-22.

[39] R. Tian, L. Wen, Improved XFEM-An extra-dof free, well- conditioning, and interpolating XFEM, Comp. Methods Appl. Mech. Engrg. 285 (2015) 639-658.

[40] L. Wen, R. Tian, An extra dof-free and well conditioned XFEM, Proceedings of the 5th International Conference on Computational Methods. 437 (2014).

[41] J. Wu, F. Li, An improved stable XFEM (Is-XFEM) with a novel enrichment function for the computational modeling of cohesive cracks, Comp. Methods Appl. Mech. Engrg. 295 (2015) 77-107.

[42] T.P. Fries, A corrected XFEM approximation without problems in blending elements, Int. J. Numer. Methods Engrg. 75 (2008) 503-532.

[43] J. Chessa, P. Smolinski, T. Belytschko, The extended finite element method (XFEM) for solidification problems, Int. J. Numer. Methods Engrg. 53 (2002) 1959-1977.

[44] R. Grace, H.W. Wang, T. Belytschko, Blending in extended finite element method by discontinuous Galerkin and assumed strain methods, Int. J. Numer. Methods Engrg. 74 (2008) 1645-1669.

[45] L.B. Freund, Dynamic Fracture Mechanics, Cambridge University Press, Cambridge, UK, 1990.

[46] L.B. Freund. Crack propagation in an elastic solid subjected to general loading. Pt. 1. Constant rate of extension. Journal of the Mechanics and Physics of Solids. 20(1972) 129140.

[47] Y.J. Lee, L.B. Freund. Fracture initiation due to asymmetric impact loading of an edge cracked plate, J. Appl. Mech. 57 (1990) 104-111.

[48] P. Rozycki, N. Moes, E. Bechet,C, Dubois, X-FEM explicit dynamics for constant strain elements to alleviate mesh constraints on internal or external boundaries, Comp. Methods Appl. Mech. Engrg. 197 (2008) 349-363.

[49] S. Pommier, A. Gravouill, A. Combescure, N. Moes, Extended Finite Element Method for Crack Propagation. 2011, ISTE Ltd and John Wiley \& Sons, Inc.

[50] S. Mohammadi. XFEM fracture analysis of composites. 2012, John Wiley \& Sons, Ltd.

[51] J. Réthoré. Méthode éléments finis étendus en espaceet temps: application à la propagation dynamique des fissures. Ph.D. Thesis, Institut National de Sciences Appliquées de Lyon, 2005.

[52] D. Grégoire, H. Maigre, J. Réthoré, A. Combescure, Dynamic crack propagation under mixed-mode loading - Comparison between experiments and X-FEM simulations, International Journal of Solids and Structures. 44 (2007) 6517-6534.

[53] J. Rethore, A. Gravouil, A. Combescure, A stable numerical scheme for the finite element simulation of dynamic crack propagation with remeshing. Comput. Methods Appl. Mech. Engrg. 193 (2004) 4493-4510.

[54] JF. Kalthoff, Modes of dynamic shear failure in solids. Int. J. Fract. 101(2000) 1-31. 